\title{
EXTENDED SCHMIDT LAW: ROLE OF EXISTING STARS IN CURRENT STAR FORMATION
}

\author{
Yong Shi $^{1}$, George Helou $^{1}$, Lin $_{\text {Yan }}{ }^{1}$, Lee Armus $^{1}$, Yanling Wu $^{1}$, Casey Papovich $^{2}$, and Sabina Stierwalt $^{1}$ \\ ${ }^{1}$ Infrared Processing and Analysis Center, California Institute of Technology, 1200 E. California Blvd, Pasadena, CA 91125, USA \\ ${ }^{2}$ George P. and Cynthia Woods Mitchell Institute for Fundamental Physics and Astronomy, Department of Physics and Astronomy, Texas A\&M University, \\ College Station, TX 77843-4242, USA \\ Received 2010 December 8; accepted 2011 March 22; published 2011 May 9
}

\begin{abstract}
We propose an "extended Schmidt law" with explicit dependence of the star formation efficiency $\left(\mathrm{SFE}=\mathrm{SFR} / M_{\mathrm{gas}}\right)$ on the stellar mass surface density $\left(\Sigma_{\text {star }}\right)$. This relation has a power-law index of $0.48 \pm 0.04$ and a $1 \sigma$ observed scatter on the SFE of 0.4 dex, which holds over five orders of magnitude in the stellar density for individual global galaxies, including various types and especially the low-surface-brightness (LSB) galaxies that deviate significantly from the Kennicutt-Schmidt (KS) law. When applying it to regions of a sample of 12 spiral galaxies at sub-kiloparsec resolution, the extended Schmidt law not only holds for LSB regions but also shows significantly smaller scatters both within and across galaxies compared with the KS law. We argue that this new relation points to the role of existing stars in regulating the SFE, thus better encoding the star formation physics. Comparison with physical models of star formation recipes shows that the extended Schmidt law can be reproduced by some models including gas free fall in a stellar-gravitational potential and pressure-supported star formation. By implementing this new law into the analytic model of gas accretion in $\Lambda C D M$, we show that it can reproduce the observed main sequence of star-forming galaxies (a relation between the SFR and stellar mass) from $z=0$ up to $z=2$.
\end{abstract}

Key words: galaxies: evolution - galaxies: starburst - ISM: atoms - ISM: molecules - stars: formation

Online-only material: color figures, machine-readable table

\section{INTRODUCTION}

Stars form from the cold interstellar medium (ISM). The resulting stellar mass growth, chemical enrichment, and energy feedback to the ISM and intergalactic medium (IGM) are key processes of galaxy formation and evolution. Understanding how stars form is thus one of the central questions in galactic and extragalactic astronomy. Star formation invokes a series of complicated processes from gas accretion, gas cooling, and $\mathrm{H}_{2}$ formation to the final molecular cloud collapse and stellar feedback. Empirical scaling laws between star formation and gas reservoirs provide critical tests of our modeling of the above various processes and have crucial applications to studies of galaxy formation and evolution in the cosmological context.

In the pioneering work of Schmidt (1959), a simple powerlaw relation is proposed to relate the star formation rate (SFR) density to the gas density:

$$
\rho_{\mathrm{SFR}}=A \rho_{\mathrm{gas}}^{N_{\mathrm{SFR}}}
$$

where $\rho_{\mathrm{SFR}}$ is the SFR volume density, $\rho_{\mathrm{gas}}$ is the volume density of total cold gas including $\mathrm{H}$ I and molecular hydrogen $\left(\mathrm{H}_{2}\right)$, and $N_{\mathrm{SFR}}$ is the power index, while $A$ is simply assumed to be constant. Kennicutt $(1989,1998$ a) demonstrated this unambiguously in its observable form (surface density) with 61 nearby spiral galaxies and 36 infrared-selected nuclear starburst regions that span a large dynamic range and concluded that

$$
\Sigma_{\mathrm{SFR}} \propto \Sigma_{\text {gas }}^{1.4 \pm 0.15}
$$

which is often referred as the Kennicutt-Schmidt (KS) law. It makes general sense that the gas reservoir determines how many stars can form, i.e., the gas density plays the dominant role in regulating SFR. With this basic relation between SFR and gas, the star formation efficiency (SFE $=$ SFR/gas-mass in this study) follows:

$$
\mathrm{SFE}=\frac{\Sigma_{\mathrm{SFR}}}{\Sigma_{\mathrm{gas}}} \propto \Sigma_{\mathrm{gas}}^{N_{\mathrm{SFE}}}, \text { with } N_{\mathrm{SFE}}=0.4 .
$$

It should be noted that the KS law does not hold for the entire range of gas densities. An accompanying rule is the star formation threshold, introduced to explain the fact that the SFR is significantly lower than predicted by the KS law at low gas densities $\left(<\sim 1-10 M_{\odot} \mathrm{pc}^{-2}\right)$, e.g., in low-surface-brightness (LSB) galaxies or regions far outside the optical disk (e.g., Martin \& Kennicutt 2001; Wyder et al. 2009; Bigiel et al. 2008; Roychowdhury et al. 2009). Other forms of the Schmidt law ${ }^{3}$ have also been proposed, such as those that invoke the dynamical factors (Silk 1997; Elmegreen 1997; Boissier et al. 2003):

$$
\mathrm{SFE} \propto \frac{1}{\tau_{\mathrm{dyn}}},
$$

where $\tau_{\text {dyn }}$ is the orbital dynamical timescale. This relation has been demonstrated to predict the SFR as well as the KS law when considering the orbital timescale (Kennicutt 1998a), while the break may still show up at the low density end (Wyder et al. 2009).

Although the above two Schmidt relations are valid for a range of galaxy types both in the local universe (e.g., Kennicutt 1998a; Bigiel et al. 2008) and at high- $z$ (e.g., Daddi et al. 2010; Genzel et al. 2010), it is surprising that they only invoke the gas component while in reality various ISM and stellar components are intimately involved in processes of converting gas into new stars. Many of these processes are related to existing stars that form over the whole galaxy's history. For example, the

\footnotetext{
3 In this study we expand the definition of the Schmidt law to include any relationship that invokes SFRs and gas, which shares the initial idea that Schmidt proposed.
} 
Table 1

Individual Galaxies with $\mathrm{CO}$ and $\mathrm{H}$ I Data

\begin{tabular}{|c|c|c|c|c|c|c|c|c|c|c|c|}
\hline Name & $\begin{array}{l}\text { Type } \\
\text { (2) }\end{array}$ & Red & $\begin{array}{l}\text { Dist. } \\
(\mathrm{Mpc}) \\
(4)\end{array}$ & Aperture & $\begin{array}{c}\text { Area } \\
\left(\mathrm{kpc}^{2}\right) \\
(6)\end{array}$ & $\begin{array}{c}\log \left(\Sigma_{\mathrm{SFR}}\right) \\
\left(M_{\odot} \mathrm{yr}^{-1} \mathrm{kpc}^{-2}\right) \\
(7)\end{array}$ & $\begin{array}{c}\log \left(\Sigma_{\mathrm{gas}}\right) \\
\left(M_{\odot} \mathrm{pc}^{-2}\right) \\
(8)\end{array}$ & $\begin{array}{c}\operatorname{Ref}_{\text {sfr, gas }} \\
\text { (9) }\end{array}$ & $\begin{array}{c}\log \left(\Sigma_{\mathrm{star}}\right) \\
\left(M_{\odot} \mathrm{pc}^{-2}\right) \\
(10)\end{array}$ & Band $_{\text {stellar mass }}$ & $\begin{array}{r}\operatorname{Ref}_{\text {star }} \\
(12) \\
\end{array}$ \\
\hline NGC0224 & Late-type & -0.001001 & 0.78 & $R_{25}$ & 1106.29 & -3.31 & 0.81 & 1 & 2.00 & IRAC3.6 & 18 \\
\hline NGC0598 & Late-type & -0.000597 & 0.80 & $R_{25}$ & 133.25 & -2.65 & 1.16 & 1 & 1.58 & IRAC3.6 & 18 \\
\hline NGC0628 & Late-type & 0.002192 & 7.30 & $1.5 R_{25}$ & 764.54 & -2.98 & 0.90 & 2 & 1.22 & IRAC3.6 & 2 \\
\hline NGC0772 & Late-type & 0.008246 & 35.00 & $R_{25}$ & 4231.16 & -3.02 & 1.00 & 1 & 1.53 & nuv, $U, B, V, I, J, H, K$ & 18 \\
\hline
\end{tabular}

Notes. Column 1: name. Column 2: the galaxy type in this paper. Column 3: the redshift. Column 4: the distance in Mpc that is used in this work. Column 5: the definition of the aperture used to calculate the surface density of SFR, gas, and stellar mass. $R_{25}$ means the isophotal radius at 25 mag arcsec ${ }^{2}$ (usually in $B$ band). $R_{\max }$ gives the maximum extent of the galaxy at a given wavelength that is indicated in square brackets. Its value relative to $R_{25}$ is listed in parentheses whenever possible. $R_{1 / 2}$ is the half-light radius at a given wavelength that is indicated in square brackets. Column 6: the de-projected area used to derive surface densities. Column 7: the SFR surface density. Column 8: the gas surface density. Column 9: the references and therein for the SFR and gas surface density data. For those with two references, the first one is for $\mathrm{H}_{2}$ and SFR data while the second is for $\mathrm{H}$ I data. Column 10: the stellar mass surface density. Column 11: the wavelength band used to measure the stellar mass. Column 12: the references for the stellar mass data.

References. (1) Kennicutt 1998a; (2) Leroy et al. 2008; (3) Wei et al. 2010; (4) Crocker et al. 2011; (5) Wyder et al. 2009; (6) Scoville et al. 1997; (7) Graciá-Carpio et al. 2007; (8) Yun \& Scoville 1995; (9) Bryant \& Scoville 1999; (10) Yun et al. 1994; (11) Genzel et al. 2010; (12) Oosterloo et al. 2010; (13) Irwin et al. 1987; (14) van Driel \& van Woerden 1991; (15) Sage \& Welch 2006; (16) Knapp \& Raimond 1984; (17) Cox et al. 2001; (18) This work.

(This table is available in its entirety in a machine-readable form in the online journal. A portion is shown here for guidance regarding its form and content.)

gravitational effects of stellar bars can remove gas angular momentum and increase nuclear SFRs in normal galaxies (e.g., Sérsic \& Pastoriza 1967; Ho et al. 1997). Their effects are also recognized in numerical simulations of gas-rich galaxy merging and thought to be the main factor to determine how much gas can be converted into stars (Hopkins et al. 2009a, 2009b). The gas hydrostatic pressure produced by gas self-gravity and stellar gravity is further shown empirically to be related to the $\mathrm{H}_{2}$-toH I ratio (Elmegreen \& Parravano 1994; Wong \& Blitz 2002; Blitz \& Rosolowsky 2004, 2006). Besides the stellar gravity, the metal outputs of stellar evolution are the main coolants of gas and thus star formation should show dependences on the metallicity. Theoretical works have indicated significantly lower SFEs at low metallicity (Krumholz et al. 2009; Gnedin \& Kravtsov 2010). Dust grains that form from metals catalyze the $\mathrm{H}_{2}$ formation and shield it from radiation destruction (for a review, see Hollenbach \& Tielens 1999). Current proposed Schmidt relations do not hint at any of these effects, as they only invoke the gas component.

In evaluating the importance of existing stars in the empirical scaling law, we demonstrate the existence of a tight relationship between SFEs and stellar mass surface densities, referred to as the extended Schmidt law. This relation not only predicts the SFE and SFR as well as the KS law for galaxies and spatially resolved regions ( $\sim 1 \mathrm{kpc}$ sizes) where the KS law works, but also holds for LSB galaxies and regions where the KS law fails. Similar close links between star formation and total stars have been recognized in previous works for certain galaxy types or limited stellar mass surface density ranges. For example, Hunter et al. (1998) have shown for radial azimuthally averaged quantities in LSB galaxies that the stellar mass density is the only quantity spatially related to the SFR density. Similar close associations between stellar masses and SFR densities are also seen within and among galaxies by Ryder \& Dopita (1994), Brosch et al. (1998), Hunter \& Elmegreen (2004), and recently by Leroy et al. (2008) for specific galaxy types or limited density ranges. Here, we demonstrate this intimate association more generally by showing a tight relation between the SFE and stellar mass density over a large dynamic range. In Section 2, we present the sample selection and data collection. We show the result in Section 3. In Section 4, we first compare the extended Schmidt law to the model of the star formation recipe and then discuss its implication for the main sequence of star-forming galaxies. Conclusions are presented in Section 5. Throughout this paper, we assume $H_{0}=70 \mathrm{~km} \mathrm{~s}^{-1} \mathrm{Mpc}^{-1}, \Omega_{0}=0.3$, and $\Omega_{\Lambda}=0.7$.

\section{SAMPLE AND DATA}

\subsection{Individual Global Galaxies}

Our whole sample is listed in Table 1 and composed of five sub-samples including low-redshift late-type galaxies, early-type galaxies, LSB galaxies, luminous infrared galaxies (LIRGs), and high- $z$ objects. The $\mathrm{CO}$ and $\mathrm{H}$ I data are collected from the literature. Molecular gas masses are derived from the $\mathrm{CO}$ by assuming a constant CO-to- $\mathrm{H}_{2}$ conversion factor of $X_{\mathrm{CO}}=2.0 \times 10^{20} \mathrm{~cm}^{-2}\left(\mathrm{~K} \mathrm{~km} \mathrm{~s}^{-1}\right)^{-1}$ or $3.17 M_{\odot} \mathrm{pc}^{-2}$ $\left(\mathrm{K} \mathrm{km} \mathrm{s}^{-1}\right)^{-1}$ or $7845 d_{\mathrm{L}}^{2} M_{\odot}(\mathrm{Jy} \mathrm{km} \mathrm{s})^{-1}$ where $d_{\mathrm{L}}$ is the luminosity distance in Mpc (Dickman et al. 1986; Solomon et al. 1987; Tacconi et al. 2008). Different conversion factors for mergers and non-mergers are also discussed in Section 3.1. A factor of 1.36 is further included to account for the presence of heavier elements in both the $\mathrm{H}_{2}$ and $\mathrm{H}_{\mathrm{I}}$ masses. The SFR data are collected from the same references as the gas data (see Table 1), and all are corrected to Chabrier initial mass function $(\mathrm{IMF})$ where $\mathrm{SFR}_{\text {Chabrier }} \approx \mathrm{SFR}_{\text {Kroupa }}=0.66 \mathrm{SFR}_{\text {Salpeter }}$ (Bruzual \& Charlot 2003).

The stellar masses are measured by fitting the Bruzual \& Charlot (2003) population synthesis model to the multi-band spectral energy distribution (SED) with Chabrier IMF following Shi et al. (2008). The details of the parameters to produce the models are listed in Table 2. The model of Bruzual \& Charlot (2003) generally produces consistent color and mass-to-light ratio at various bands compared to others (Vázquez \& Leitherer 2005). However, it still lacks accurate evolutionary tracks of thermally pulsing asymptotic giant branch (AGB) stars. At the stellar age around 0.1-3 Gyr where AGB stars are prominent, the mass-to-light ratio in the near-IR can be overestimated by $\sim 60 \%$ (Maraston et al. 2006; Bruzual 2007). The effect of this on the result of this paper is shown to be small (see Section 3.1). To minimize systematic errors that may be caused by different template population models, the range of input parameter spaces and numerical method, we have applied the above method to all of our objects except for 23 galaxies from Leroy et al. (2008) 
Table 2

The Parameters of Stellar Synthesis Models

\begin{tabular}{ll}
\hline \hline \multicolumn{1}{c}{ Parameters } & \multicolumn{1}{c}{ Value } \\
\hline Simple stellar populations & Chabrier (2003) IMF and Padova 1994 evolutionary tracks \\
Metallicity & $0.0004,0.004,0.008,0.02\left(Z_{\odot}\right), 0.05$ \\
Visual extinction $\tau_{v}$ & {$[0.0,10.0]$ with a step of 1} \\
Fraction of $\tau_{V}$ arising from the ambient ISM & 0.3 \\
Fraction of ejected gas to be recycled in stars & 0.0 \\
Star formation history (SFH) & Exponential decline \\
$e$-folding time $\tau$ for exponential SFH & {$[0.03,22.4]$ Gyr with a step of 0.15 in logarithm, plus $100 \mathrm{Gyr}$} \\
Galaxy age & {$[0.05,12.6]$ Gyr with a step of 0.12 in logarithm }
\end{tabular}

whose masses are derived from IRAC $3.6 \mu \mathrm{m}$ emission in that work. For these objects, the published gas and SFR surface densities are defined within $1.5 R_{25}$ where the available optical photometry does not exist. For the spiral objects in their sample where the majority (>95\%) of the optical light is enclosed within $R_{25}$ (Courteau 1996), the median offset between their stellar masses and ours by fitting models to optical/IRAC photometry is only 0.1 dex. The broadband wavelengths used to calculate the stellar masses for all objects are listed in Table 1 and the majority covers both the optical and near-IR bands.

The surface densities of gas, SFRs, and stellar masses are measured within the same aperture and corrected for inclination. Table 1 lists the aperture definitions and their relations to optical isophotal radii $\left(R_{25}\right)$ if available in Paturel et al. (2003). ${ }^{4}$ Different apertures are adopted for different galaxy types, not only because of the heterogeneous nature of the sample but also because of different light structures of individual galaxy types. Since a star formation law describes how star forms from gas, an ideal aperture for a galaxy-averaged star formation relationship should enclose the majority of star formation or gas. This certainly results in different aperture sizes for galaxies with different types, e.g., compact apertures for ultraluminous infrared galaxies (ULIRGs) and wide ones for LSB galaxies. As discussed in Section 3.1, the extended Schmidt law depends little on how exactly an aperture is defined. We estimated a typical error of 0.30 dex for each quantity (SFR, gas, and stellar mass densities) based on our own experiences of measurements, while local LIRGs and high- $z$ galaxies have higher uncertainties ( $\sim 0.5 \mathrm{dex})$ due to low spatial resolution.

1. Late-type galaxies. This sub-sample includes 61 and 18 objects from Kennicutt (1998a) and Leroy et al. (2008), respectively. For 11 objects included in both samples, the data from the latter are used in this work because of higher data quality. Galaxies from Kennicutt (1998a) have published SFR and gas surface densities that are defined to be within the optical isophotal radii $\left(R_{25}\right.$; de Vaucouleurs et al. 1976). The SFR is based on the extinction-corrected $\mathrm{H} \alpha$ emission with $A_{\mathrm{H} \alpha}=1.1 \mathrm{mag}$ (see their paper for the equation). The optical/near-IR photometry used to derive stellar masses are collected from NED. Since the aperture of $R_{25}$ encloses the majority ( $>95 \%$ ) of optical light for spiral galaxies (Courteau 1996), the aperturematched stellar mass density is thus defined by dividing the total mass with $\pi R_{25}^{2}$. All spiral galaxies from Leroy et al. (2008) have published gas, SFR, and stellar mass densities, where the aperture radius is defined to be $1.5 R_{25}$. The SFR is measured from the combination of FUV and $24 \mu \mathrm{m}$ emission (see their paper for the equation). For 11 objects from this study that are also included in the sample

\footnotetext{
4 http://leda.univ-lyon1.fr/
}

of Kennicutt (1998a), we checked the median offset in the stellar mass between ours and theirs is only 0.1 dex.

2. Early-type galaxies. Low level star formation has been detected in circumnuclear regions of many early-type galaxies (Shapiro et al. 2010; Wei et al. 2010; Crocker et al. 2011). Besides the difficulty of SFR measurements due to contamination from large populations of old stars and possible active galactic nucleus (AGN), these objects are shown to follow more or less the KS law. We here included nine objects from Wei et al. (2010) and 10 objects from Crocker et al. (2011). Wei et al. (2010) have published the aperture-matched $\mathrm{H}_{2}$ mass from $\mathrm{CO}$ data and the SFR from a combination of $24 \mu \mathrm{m}$ and UV emission following Leroy et al. (2008). For six of nine objects, we estimated the H I mass from the available Very Large Array (VLA) H I map in the literature (see Table 1) and found that the H I mass in the $\mathrm{CO}$ aperture is $<10 \%$ of the total H I. Based on this, we therefore included three more objects with the total $\mathrm{H} \mathrm{I}$ mass $<1.6$ times the $\mathrm{H}_{2}$ mass, which implies the $\mathrm{H}$ I mass within the $\mathrm{CO}$ aperture is smaller than $16 \%$ of $\mathrm{H}_{2}$. Objects in Crocker et al. (2011) have available aperture-matched total gas mass and SFR measurements. As recommended in that work, we have used $24 \mu \mathrm{m}+\mathrm{H} \alpha$ or $\mathrm{PAH}+\mathrm{H} \alpha$ if available and radio $+\mathrm{H} \alpha$ for two more objects, where they have used formula calibrated in Kennicutt et al. (2009, see their Table 4). For objects from both works, the aperture-matched stellar mass density is estimated in this work by fitting the stellar synthesis models to the aperture-matched Two Micron All Sky Survey (2MASS) $J, H$, and $K$ photometry and Sloan Digital Sky Survey (SDSS) optical photometry for most of them.

3. LSB galaxies. We defined the LSB objects as an independent subsample, as they show deviations from the KS law. The subsample includes all the 19 objects from Wyder et al. (2009) and five gas-rich dwarf galaxies from Leroy et al. (2008) that have stellar mass surface densities below $1 M_{\odot} \mathrm{pc}^{-2}$, which is roughly the upper limit of the Wyder et al. (2009) sample. Wyder et al. (2009) measured the surface densities of the SFR and gas mass through UV emission (using the Kennicutt $1998 \mathrm{~b}$ equation) and $\mathrm{H}$ I, respectively, where the aperture is defined to be the minimum of the maximum radii of UV and $\mathrm{H}$ I emission. For these objects, the contribution from obscured star formation should be negligible to the total SFR (e.g., Hinz et al. 2007), while the H I should dominate the total gas mass (e.g., Matthews et al. 2005; Leroy et al. 2008). We derived the stellar mass from the SDSS photometry, while additional near-IR photometry from NED was also used for two objects. As the published SDSS data underestimate the sizes and thus brightnesses of these objects, we have re-measured five-band SDSS photometry in the aperture adopted in Wyder et al. (2009). The 
Table 3

The Sample of Spiral Galaxies

\begin{tabular}{|c|c|c|c|c|c|c|}
\hline $\begin{array}{c}\text { Name } \\
(1) \\
\end{array}$ & $\begin{array}{c}\text { Position (J2000) } \\
(2) \\
\end{array}$ & $\begin{array}{l}\text { Type } \\
\text { (3) }\end{array}$ & $\begin{array}{c}\text { Dist } \\
(\mathrm{Mpc}) \\
(4) \\
\end{array}$ & $\begin{array}{c}R_{25} \\
(\operatorname{arcsec}) \\
(5)\end{array}$ & $\begin{array}{l}i \\
(0) \\
(6) \\
\end{array}$ & $\begin{array}{l}\text { P.A. } \\
(0) \\
(7) \\
\end{array}$ \\
\hline NGC0628 & $013641.8+154700$ & SAc & 7.30 & 293.17 & 7.00 & 20.00 \\
\hline NGC2841 & $092202.6+505835$ & $\mathrm{SAb}$ & 14.10 & 207.55 & 74.00 & 153.00 \\
\hline NGC3184 & $101817.0+412528$ & SABcd & 11.10 & 222.39 & 16.00 & 179.00 \\
\hline NGC3351 & $104357.7+114214$ & $\mathrm{SBb}$ & 10.10 & 217.33 & 41.00 & 192.00 \\
\hline NGC3521 & $110548.6-000209$ & $\mathrm{SABbc}$ & 10.70 & 249.53 & 73.00 & 340.00 \\
\hline NGC3627 & $112015.0+125930$ & $\mathrm{SABb}$ & 9.30 & 306.99 & 62.00 & 173.00 \\
\hline NGC4736 & $125053.0+410713$ & SAab & 4.70 & 232.87 & 41.00 & 296.00 \\
\hline NGC4826 & $125643.6+214100$ & SAab & 7.50 & 314.14 & 65.00 & 121.00 \\
\hline NGC5055 & $131549.2+420145$ & $\mathrm{SAbc}$ & 10.10 & 352.47 & 59.00 & 102.00 \\
\hline NGC5194 & $132952.7+471143$ & $\mathrm{SABbc}$ & 8.00 & 232.87 & 42.00 & 172.00 \\
\hline NGC6946 & $203452.2+600914$ & SABcd & 5.90 & 344.45 & 33.00 & 243.00 \\
\hline NGC7331 & $223704.1+342457$ & $\mathrm{SAb}$ & 14.70 & 273.60 & 76.00 & 168.00 \\
\hline
\end{tabular}

Notes. Column 1: name; Column 2: the position; Column 3: the Hubble type; Column 4: the distance; Column 5: the optical size $R_{25}$; Column 6: the inclination; Column 7: the position angle.

majority of these objects have optical emission out to the adopted radius as observed by SDSS (see the $r$-band radial profile in Wyder et al. 2009). The comparison of our $r$-band photometry to those measured by Wyder et al. (2009) shows $<10 \%$ discrepancy. All dwarf galaxies from Leroy et al. (2008) have published gas, SFR, and stellar mass densities within $1.5 r_{25}$.

4. Local LIRGs $(z=0$ LIRGs). We have collected seven local LIRGs from the literature with two criteria: (a) the spatially resolved $\mathrm{CO}$ interferometer images are available and well resolved (i.e., the deconvolved size is larger than the resolution); (b) the interferometer fluxes recover the majority $(>90 \%)$ of single-dish measurements. As listed in Table 1, the aperture to define the surface density is the maximum extent of the $\mathrm{CO}$ emission. For the total gas density, we have neglected the contribution from the atomic gas in these gas-rich objects. Since star formation takes place in molecular clouds, we assume all star formation is included in the CO aperture where the SFR is based on the IR luminosity assuming the Kennicutt (1998b) relations and corrected to Chabrier IMF. To measure the aperturematched stellar mass density, we first calculated the total mass by fitting stellar models to the UV/optical/near-IR SED collected from NED, and then measured the part in the $\mathrm{CO}$ aperture with the HST/ACS-F814w image assuming a constant mass-to-light ratio, which can cause additional stellar mass uncertainty ( $\sim 0.3 \mathrm{dex})$.

5. High-redshift star-forming galaxies (high-z SFGs) and merging submillimeter galaxies (high-z SMGs). We here included 21 optically/near-IR selected star-forming galaxies (Extended Groth Strip (EGS), BzK, and BXMD) and seven submillimeter objects from Genzel et al. (2010). The SFR and gas are available in that work. The SFR for the EGS is estimated from a combination of extinction-corrected $\mathrm{H} \alpha /[\mathrm{O} \mathrm{II}] / G A L E X-\mathrm{UV}$ and Spitzer $24 \mu \mathrm{m}$ emission. For the $\mathrm{BzK}$, it is a combination of extinction-corrected UV and $24 \mu \mathrm{m}$ emission. For the BXMD objects, it is from extinction-corrected $\mathrm{H} \alpha$ emission while the SFR of the SMG is from $850 \mu \mathrm{m}$ emission (see Genzel et al. 2010 for the equation). We re-derived stellar mass estimates to reduce systematic errors among different studies. The median offsets of our stellar masses compared to the literature data are $-0.16 \mathrm{dex}, 0.34 \mathrm{dex},-0.55 \mathrm{dex}$, and 0.00 dex for EGS,
BzK, BXMD, and submillimeter galaxies, respectively (see Genzel et al. 2010, Daddi et al. 2010, Erb et al. 2006, and Hainline et al. 2010, respectively). The large offsets for BXMD $(z \sim 2.2)$ and BzK $(z \sim 1.5)$ are mainly due to the lack of the rest-frame near-IR photometry, which could cause large differences when different template SEDs are used. With the unified stellar mass measurement, the scatter among them in the extended Schmidt law does become smaller. The galaxy size is defined as the half-light radius obtained from the fit to $\mathrm{H} \alpha$, optical/UV, or CO images. Similar to Genzel et al. (2010), all densities are defined within the half-light radius $\left(R_{1 / 2}\right)$, e.g., $\Sigma_{\text {star }}=0.5 M_{\text {star }} /$ $\left(\pi R_{1 / 2}^{2}\right)$. Here we did not account for the difference in the half-light radius among SFR, gas, and stars for individual galaxies, since these three half-light radii are on average quite close to each other (e.g., Swinbank et al. 2010).

\subsection{Individual Regions in Spiral Galaxies}

With the advent of high spatial-resolution SFR, gas, and stellar images of nearby galaxies, the star formation law at sub-kiloparsec scales has been studied extensively (e.g., Wong \& Blitz 2002; Jogee et al. 2005; Crosthwaite \& Turner 2007; Schuster et al. 2007; Kennicutt et al. 2007; Bigiel et al. 2008; Leroy et al. 2008). The general conclusion is that the relationship between SFR and total gas varies strongly both within galaxies and across different objects. This implies that the physics other than those directly related to the total gas strongly affect the SFE of the total gas. To test the idea of the SFE regulated by existing stars as proposed by the extended Schmidt law, we have carried out measurements of SFR, gas, and stellar mass in a sample of 12 spiral galaxies as listed in Table 3. They are derived from The H I Nearby Galaxy Survey (THINGS; Walter et al. 2008) and The SIRTF Nearby Galaxies Survey (SINGS; Kennicutt et al. 2003).

For each object, the SFR and gas mass are measured within individual $750 \times 750 \mathrm{pc}^{2}$ regions across the main optical disk (semimajor axis $<R_{25}$ ), with the technical procedure basically following Bigiel et al. (2008, also see Leroy et al. 2008) but corrected to our IMF and CO-to- $\mathrm{H}_{2}$ conversion factors. The SFR is estimated from combination of GALEX far-UV (Gil de Paz et al. 2007) and Spitzer $24 \mu \mathrm{m}$ (Kennicutt et al. 2003), with the $3 \sigma$ lower limit around $10^{-4} M_{\odot} \mathrm{yr}^{-1} \mathrm{kpc}^{-2}$. The gas mass is 


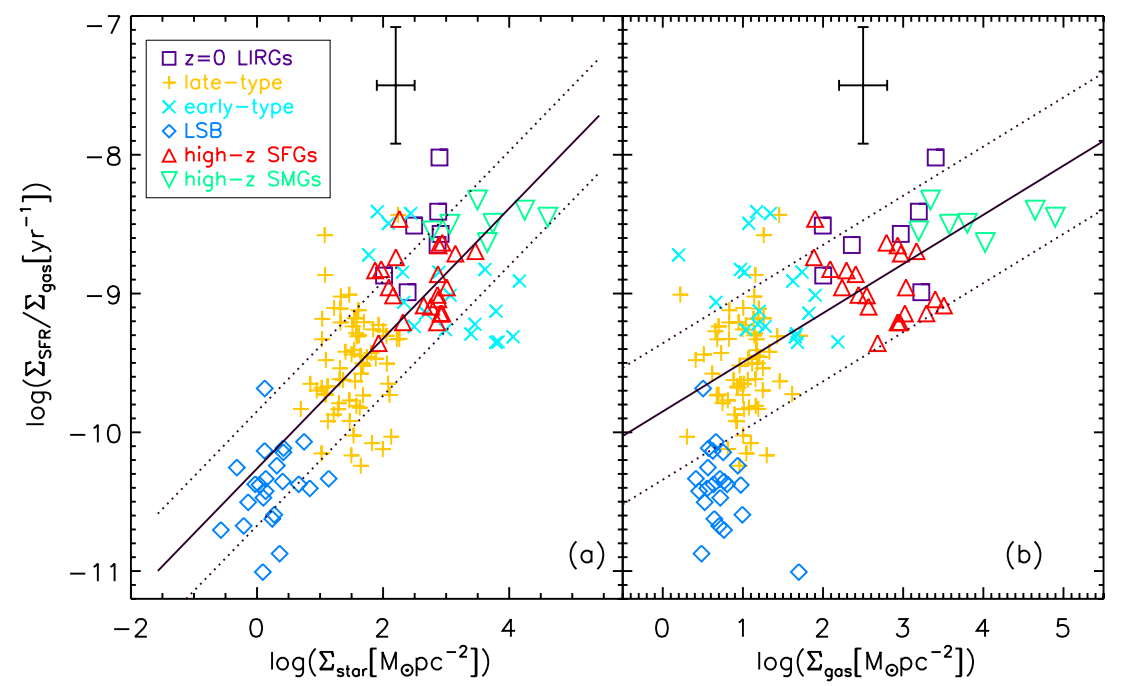

Figure 1. (a) The star formation efficiency $\left(=\Sigma_{\mathrm{SFR}} / \Sigma_{\text {gas }}\right)$ as a function of the stellar mass surface density. (b) The SFE as a function of the gas density. The solid and dotted lines are the intrinsic best-fit and observed $1 \sigma$ scatter, respectively, as listed in Table 4 . The fit to the SFE- $\Sigma_{\text {gas }}$ (right panel) is done excluding LSB and early-type galaxies. Typical measurement error bars are plotted.

(A color version of this figure is available in the online journal.)

derived from a combination of THINGS H I data (Walter et al. 2008) and BIMA SONG CO $J=1-0$ map (Helfer et al. 2003), with the limiting surface density around $1.5 M_{\odot} \mathrm{pc}^{-2}$. Since the spatial coverage of BIMA SONG does not extend significantly beyond the $\mathrm{HI}$-to- $\mathrm{H}_{2}$ transition radius where the total gas is dominated by $\mathrm{H}$ I emission, we have extrapolated the $\mathrm{CO}$ data to pixels without observations based on the observed $\mathrm{CO} / \mathrm{H}$ I ratio as a function of semimajor axis. The result changes little if adopting the mean $\mathrm{CO} / \mathrm{H}$ I ratio as a function of radius derived from Leroy et al. (2008). For seven galaxies with significantly extended CO coverage in Bigiel et al. (2008), our derived slope of the KS law is consistent with theirs within $20 \%$. The stellar mass is estimated based on the mass-to-light ratio at Spitzer $3.6 \mu \mathrm{m}$. The SINGS $3.6 \mu \mathrm{m}$ image is further subtracted by the median sky level after binning to the resolution of $750 \times 750 \mathrm{pc}^{2}$, while the $3 \sigma$ sky fluctuation gives the limiting stellar mass surface density around $1 M_{\odot} \mathrm{pc}^{-2}$. To reduce the effect of the radial gradient of stellar age, extinction, and metallicity on the stellar mass measurement, the $3.6 \mu \mathrm{m}$ mass-to-light ratio is derived based on the optical color, for which the theoretical trend is computed from our stellar population synthesis models with solar and 0.25 solar metallicity. For five galaxies with available SDSS images, the optical color is defined to be the $g-r$ color, while the mean trend of these objects is used for the remaining objects.

\section{RESULTS}

\subsection{The Extended Schmidt Law for Individual Galaxies}

By treating $\Sigma_{\mathrm{SFR}}$ as a dependent variable, and $\Sigma_{\text {gas }}$ and $\Sigma_{\text {star }}$ as independent variables, we search for the best powerlaw exponents relating them. A linear regression fit (IDL regress.pro) gives

$$
\Sigma_{\mathrm{SFR}} \propto \Sigma_{\text {gas }}^{1.13 \pm 0.05} \Sigma_{\text {star }}^{0.36 \pm 0.04} .
$$

The most important result of the fit is that the derived index for $\Sigma_{\text {star }}$ is not zero, which would be expected by the KS law. Second, the derived exponent for $\Sigma_{\text {gas }}$, namely, approximately unity, suggests a clear physical implication of the relation, i.e., that the SFE $\left(=\Sigma_{\mathrm{SFR}} / \Sigma_{\mathrm{gas}}\right)$ is related to the stellar mass surface density. We thus carry out directly the fit between SFE and $\Sigma_{\text {star }}$ as shown in Figure 1(a) through a Bayesian approach to linear regression (Kelly 2007) that also accounts for uncertainties in both variables. As shown in Table 4, the best fit gives

$$
\frac{\mathrm{SFE}}{\mathrm{yr}^{-1}}=10^{-10.28 \pm 0.08}\left(\frac{\Sigma_{\text {star }}}{M_{\odot} \mathrm{pc}^{-2}}\right)^{0.48 \pm 0.04}
$$

where errors of the best-fit parameters are the intrinsic ones. The SFE $-\Sigma_{\text {star }}$ relation is obviously different from the KS law, although both of them could be used to predict the SFE. By invoking the stellar mass density, the SFE $-\Sigma_{\text {star }}$ correlation describes another scaling relation for star formation with emphasis on the role of the existing stellar component in the current star formation activity. In what follows, we refer to the relation in Equation (6) or the SFE- $\Sigma_{\text {star }}$ relation as the extended Schmidt law as it includes the additional parameter of stellar surface density.

We now compare the extended Schmidt law to the KS law in the ability to predict the SFE and SFR for various galaxy types as shown in Figures 1 and 2, respectively. The main difference of the extended Schmidt law from the standard KS law is to bring the LSB objects back to the relationship. While the KS law was first defined for late-type galaxies and LIRGs (Kennicutt 1989, 1998a), LSB galaxies/regions show significant deviations from it (e.g., Kennicutt 1998a; Martin \& Kennicutt 2001; Bigiel et al. 2008; Wyder et al. 2009; Roychowdhury et al. 2009). The median offsets of the LSB from the best fit are -0.75 dex and -0.71 dex in Figures 1(b) and 2(b), respectively, where the best fit is done by excluding LSB and early-type objects due to their apparent offsets. The cause for this deviation is still unclear but is most likely related to some instabilities, either gravitational or thermal and chemical. On the other hand, these LSB objects follow more or less the extended Schmidt law with median offsets of -0.16 and -0.08 dex in the two figures, respectively, where the fit is done for all objects. This is the main advantage of the extended Schmidt law compared with the KS one. The observed scatter of the extended Schmidt law is slightly smaller than that of the KS law (see Table 4). For early-type galaxies, we here included objects from studies of Wei et al. (2010) and 
Table 4

The Parameters of the Best Fit

\begin{tabular}{lcccccc}
\hline \hline $\begin{array}{c}\text { Figures } \\
(1)\end{array}$ & $\begin{array}{c}\text { Correlations } \\
(2)\end{array}$ & $\begin{array}{c}a \\
(3)\end{array}$ & $\begin{array}{c}b \\
(4)\end{array}$ & $\begin{array}{c}\delta \\
(5)\end{array}$ & $\begin{array}{c}\rho \\
(6)\end{array}$ & $\begin{array}{c}\sigma \\
(7)\end{array}$ \\
\hline Figure 1(a) & $\mathrm{SFE}-\Sigma_{\text {star }}$ & $-10.28 \pm 0.08$ & $0.48 \pm 0.04$ & 0.123 & $0.97 \pm 0.03$ & 0.41 \\
Figure 1(b) & $\mathrm{SFE}-\Sigma_{\text {gas }}$ & $-9.85 \pm 0.08$ & $0.35 \pm 0.04$ & 0.092 & $0.97 \pm 0.03$ & 0.49 \\
Figure 2(a) & $\Sigma_{\mathrm{SFR}}-\Sigma_{\text {star }}^{0.5} \Sigma_{\text {gas }}$ & $-4.40 \pm 0.08$ & $1.03 \pm 0.03$ & 0.131 & $1.00 \pm 0.00$ & 0.42 \\
Figure 2(b) & $\mathrm{KS}$ law & $-3.90 \pm 0.11$ & $1.38 \pm 0.06$ & 0.112 & $1.00 \pm 0.00$ & 0.49 \\
Figure 3(a) & $\mathrm{SFE}-\Sigma_{\text {star }}$ (diff. $\left.\alpha\right)$ & $-10.35 \pm 0.09$ & $0.55 \pm 0.04$ & 0.171 & $0.96 \pm 0.03$ & 0.47 \\
Figure 3(b) & $\mathrm{SFE}-\Sigma_{\text {gas }}$ (diff. $\left.\alpha\right)$ & $-9.97 \pm 0.10$ & $0.49 \pm 0.06$ & 0.172 & $0.93 \pm 0.05$ & 0.54 \\
Figure 3(c) & $\Sigma_{\text {SFR }}-\Sigma_{\text {sta }}^{0.5} \Sigma_{\text {gas }}($ diff. $\alpha)$ & $-4.51 \pm 0.09$ & $1.10 \pm 0.03$ & 0.180 & $0.99 \pm 0.01$ & 0.47 \\
Figure 3(d) & $\mathrm{KS} \mathrm{law} \mathrm{(diff.} \alpha)$ & $-4.17 \pm 0.14$ & $1.65 \pm 0.09$ & 0.125 & $1.00 \pm 0.00$ & 0.55 \\
\hline
\end{tabular}

Notes. Column 1: the corresponding figure to a given correlation. Note that the LSB and early-type galaxies are always excluded for the KS law or the equivalent version $\left(\mathrm{SFE}-\Sigma_{\mathrm{gas}}\right)$. "diff. $\alpha$ " means different CO-to- $\mathrm{H}_{2}$ conversion factors for mergers and non-mergers. Column 2: the $Y$ vs. $X$ correlations. Column 3-5: $\log (Y)=a+$ $b * \log (X)+\epsilon$ as given by linmix_err.pro that accounts for measured errors on both $X$ - and $Y$-axis (Kelly 2007), where $(a, b)$ are the regression coefficients and $\epsilon$ is the intrinsic random scatter about the regression and has a mean of zero and variance of $\delta^{2}$. Column 6: the linear correlation efficiency. Column 7: the observed standard deviation of all data points from the best fit.

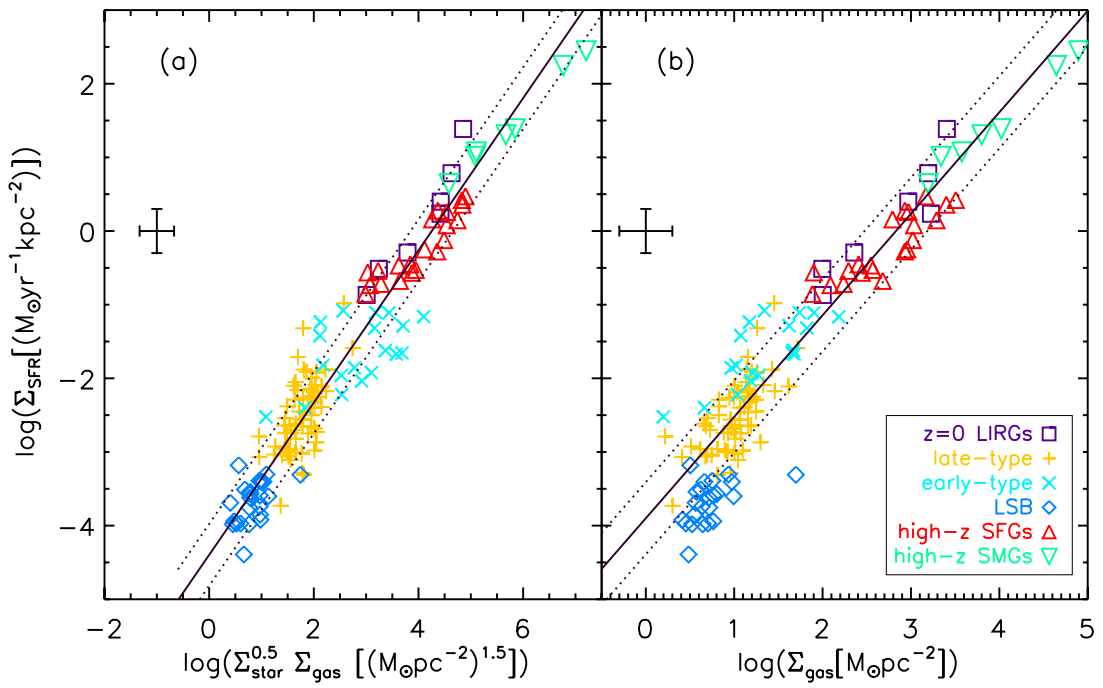

Figure 2. Comparison between the extended Schmidt law and Kennicutt-Schmidt relationship in the ability to predict the SFR. The solid and dotted lines are the intrinsic best-fit and observed $1 \sigma$ scatter, respectively, as listed in Table 4. The fit to the KS law (right panel) is done excluding LSB and early-type galaxies. Typical measurement error bars are plotted.

(A color version of this figure is available in the online journal.)

Crocker et al. (2011). They follow more or less the KS law with median offsets of 0.30 dex and 0.32 dex toward higher SFE (Figure 1(b)) and SFR (Figure 2(b)), respectively. The objects from Wei et al. (2010) show slightly larger offsets than Crocker et al. (2011), probably due to the contribution from old stellar populations to the UV emission that has been included in their SFR measurements. As shown in Figures 1(a) and 2(a), these early-type galaxies lie generally on the extended Schmidt law with median offsets of -0.19 dex and -0.26 dex on two figures, respectively. As a summary, we found that the extended Schmidt law is a universal relationship that holds for various galaxy types especially including LSB ones that do not follow the KS law.

As described in Section 2.1, different galaxy apertures are used for different galaxy types. As listed in Table 1, their relative sizes to optical isophotal radii $R_{25}$ range from around $0.2 R_{25}$ for LIRGs and early-type galaxies with circumnuclear star formation to $2-3 R_{25}$ for LSB galaxies with widely distributed star formation. Most of these apertures are indeed defined to enclose the majority of star formation and gas, which is consistent with the definition of star formation law that empirically describes how stars form gas. In spite of different apertures, the extended Schmidt law depends little on the aperture size as long as three quantities (SFR, gas, and stellar mass) are measured within the same aperture which is what has been done in this study. As shown in the below, galaxies basically move along the relation without large offsets from the best fit if different apertures are used. For 11 LSB galaxies from Wyder et al. (2009) with available radial profiles of SFR, gas, and stellar mass, we measured the relative offset in the $Y$-axis from the best fit compared to the aperture $\left(\sim 2 R_{25}\right)$ used in Figure 2 . Three apertures of $0.2 R_{25}, 0.5 R_{25}$, and $R_{25}$ are tested. As expected, they move along the relation, with median relative offsets of only $-0.02 \mathrm{dex}, 0.12 \mathrm{dex}$, and 0.04 dex, respectively, where the positive sign means offset toward higher SFRs. For 12 spiral galaxies with apertures of $1.5 R_{25}$ from Leroy et al. (2008), the median offsets for $0.5 R_{25}$ and $R_{25}$ are -0.1 dex and -0.02 dex, respectively. For early-type objects and ULIRGs, if we assume no star formation and gas outside the circumnuclear region, the median offset will be around 0.3 dex and 0.6 dex for the aperture radius of $0.5 R_{25}$ and $R_{25}$, respectively, 

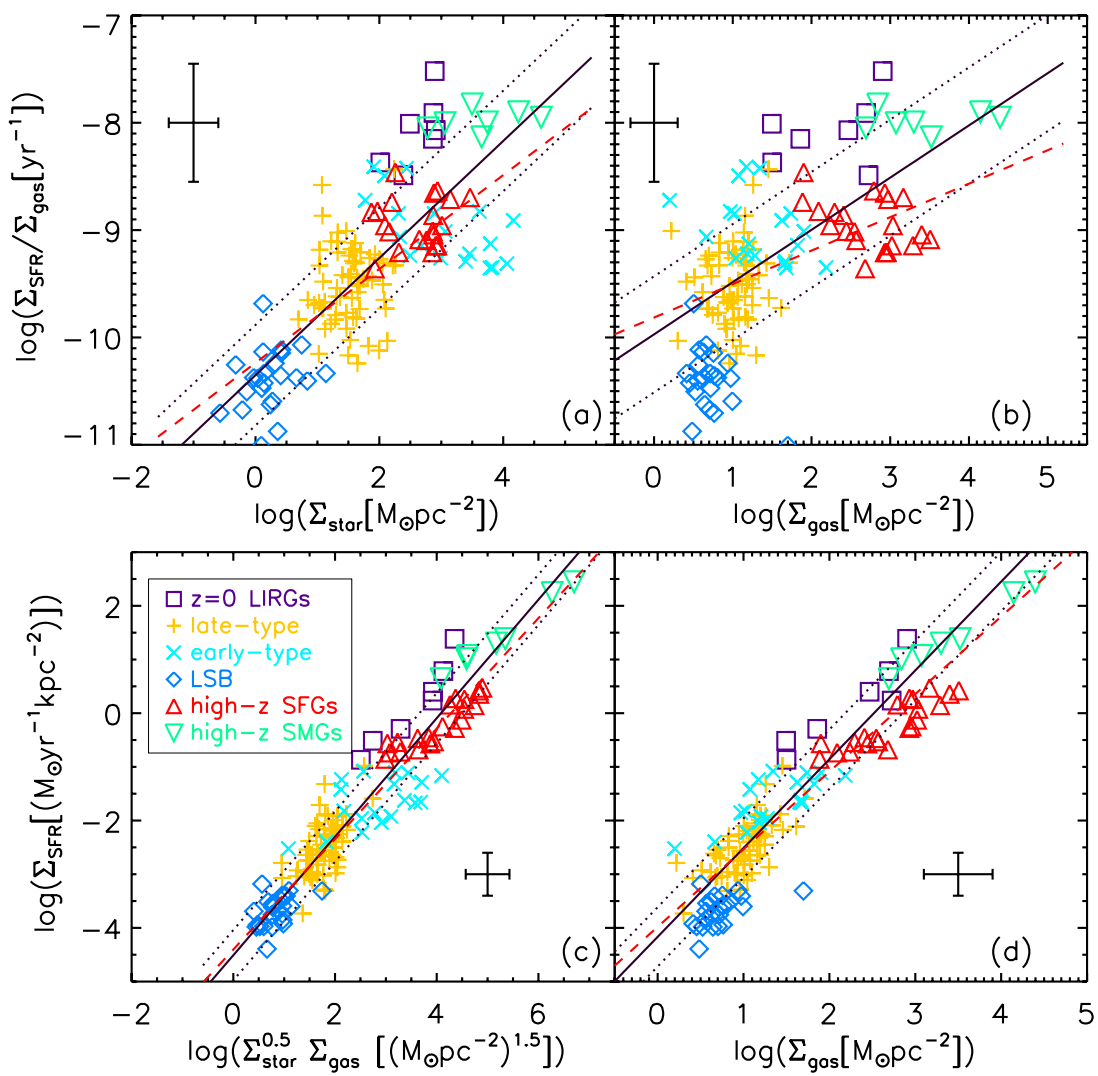

Figure 3. Similar to Figures 1 and 2 but using different CO-to- $\mathrm{H}_{2}$ conversion factors for normal galaxies $\left(\alpha=3.17 M_{\odot} \mathrm{pc}^{-2}\left(\mathrm{~K} \mathrm{~km} \mathrm{~s}^{-1}\right)^{-1}\right)$ and merging objects $($ local LIRGs and high- $z$ submillimeter galaxies; $\left.\alpha=1.0 M_{\odot} \mathrm{pc}^{-2}\left(\mathrm{~K} \mathrm{~km} \mathrm{~s}^{-1}\right)^{-1}\right)$. The solid and dotted lines are the intrinsic best-fit and observed $1 \sigma$ scatter, respectively, to all data points but excluding LSB and early-type galaxies for panels (b) and (d). The dashed lines show the best fit to all the non-mergers but excluding LSB and early-type ones for panels (b) and (d).

(A color version of this figure is available in the online journal.)

which is still within tolerance given the observed $1 \sigma$ scatter of 0.5 dex in Figure 2. Overall, we have found that the extended Schmidt law changes very little if the aperture varies by a factor of 3-5.

Accurate stellar mass measurements are important to the result of the extended Schmidt law. To reduce systematic errors by different studies, we have measured the masses on our own based on the Bruzual \& Charlot (2003) model for almost all objects except for 23 galaxies in Leroy et al. (2008), for which the median offset from our method is only 0.1 dex (see Section 2.1). The Bruzual \& Charlot (2003) model underestimates the contribution from AGB stars in the nearIR and thus overestimates the near-IR mass-to-light ratio at ages around 0.1-3 Gyr. If the stellar masses are reduced by $60 \%$ for galaxies with characteristic ages defined by stellarmass/SFR around the above range, the slope of the extended Schmidt law increases only by $\sim 0.01 \mathrm{dex}$. As listed in Table 2, we adopted exponentially declining or constant star formation history (SFH). If the SFH is exponentially increasing for the high- $z$ objects (Maraston et al. 2010; Papovich et al. 2011), the inferred stellar mass decreases by about 0.2 dex and the slope of the extended Schmidt law only increases by $0.01 \mathrm{dex}$. We notice that the photometric coverage of LSB galaxies is generally not as good as other types. While those from Wyder et al. (2009) are essentially based on the SDSS optical photometry, the ones from Leroy et al. (2008) are based on IRAC $3.6 \mu \mathrm{m}$. The median offset in the stellar mass density between the two subsample is only 0.04 dex, implying that there is no significant bias in the mass estimate based only on either optical or near-IR photometry.
Recently, Schiminovich et al. (2010) published the HIbased SFE measurement for a large sample of local massive galaxies $\left(M_{*}>10^{10} M_{\odot}\right)$. They claimed an almost constant SFE $\left(10^{-9.5} \mathrm{yr}^{-1}\right)$ in their sample for the range of $\log \left(\Sigma_{\text {star }}\right)$ of 2-3.3 $M_{\odot} \mathrm{kpc}^{-2}$. The detailed comparison to our result is hampered by the lack of the $\mathrm{H}_{2}$ data for their objects, possible large uncertainties in their SFR measurements (UV-based ones versus our recombination-line/IR/UV-based ones) and most importantly the aperture-unmatched SFE measurements relative to those of the stellar densities measured within the half-light radius $\left(r_{1 / 2}\right)$. To estimate a rough deviation of their sample from the prediction of our relation given their median density, we assume $M_{\mathrm{HI}}+\mathrm{H}_{2} / M_{\mathrm{HI}} \approx 10$ within $r_{1 / 2}$ (Leroy et al. 2008) and $M_{\mathrm{HI}}^{\mathrm{tot}} / M_{\mathrm{HI}}^{r_{1 / 2}} \approx 50$ derived from the THINGS H r radial profile in Walter et al. (2008). The resulting offset is $<0.3 \mathrm{dex}$.

The above studies assume the same CO-to- $\mathrm{H}_{2}$ factor $(\alpha$ value) for all galaxies, whereas this factor is likely to be different in local ULIRGs and high- $z$ merging galaxies from normal galaxies. To quantify the effect of different $\alpha$ values in the relation, we show the relations in Figure 3 with $\alpha=$ $3.17 M_{\odot} \mathrm{pc}^{-2}\left(\mathrm{~K} \mathrm{~km} \mathrm{~s}^{-1}\right)^{-1}$ for normal galaxies (Dickman et al. 1986; Solomon et al. 1987; Tacconi et al. 2008) and $\alpha=1.0 M_{\odot} \mathrm{pc}^{-2}\left(\mathrm{~K} \mathrm{~km} \mathrm{~s}^{-1}\right)^{-1}$ for merging galaxies $(z=$ 0 LIRGs and high- $z$ submillimeter galaxies in this study; Downes \& Solomon 1998; Tacconi et al. 2008). For the KS law (Figure 3(d)), the median offsets of the merging galaxies from the best fit to all data points and non-merging galaxies excluding LSB and early-type ones are 0.48 and 0.81 dex, respectively, which is consistent with the result obtained by Genzel et al. 


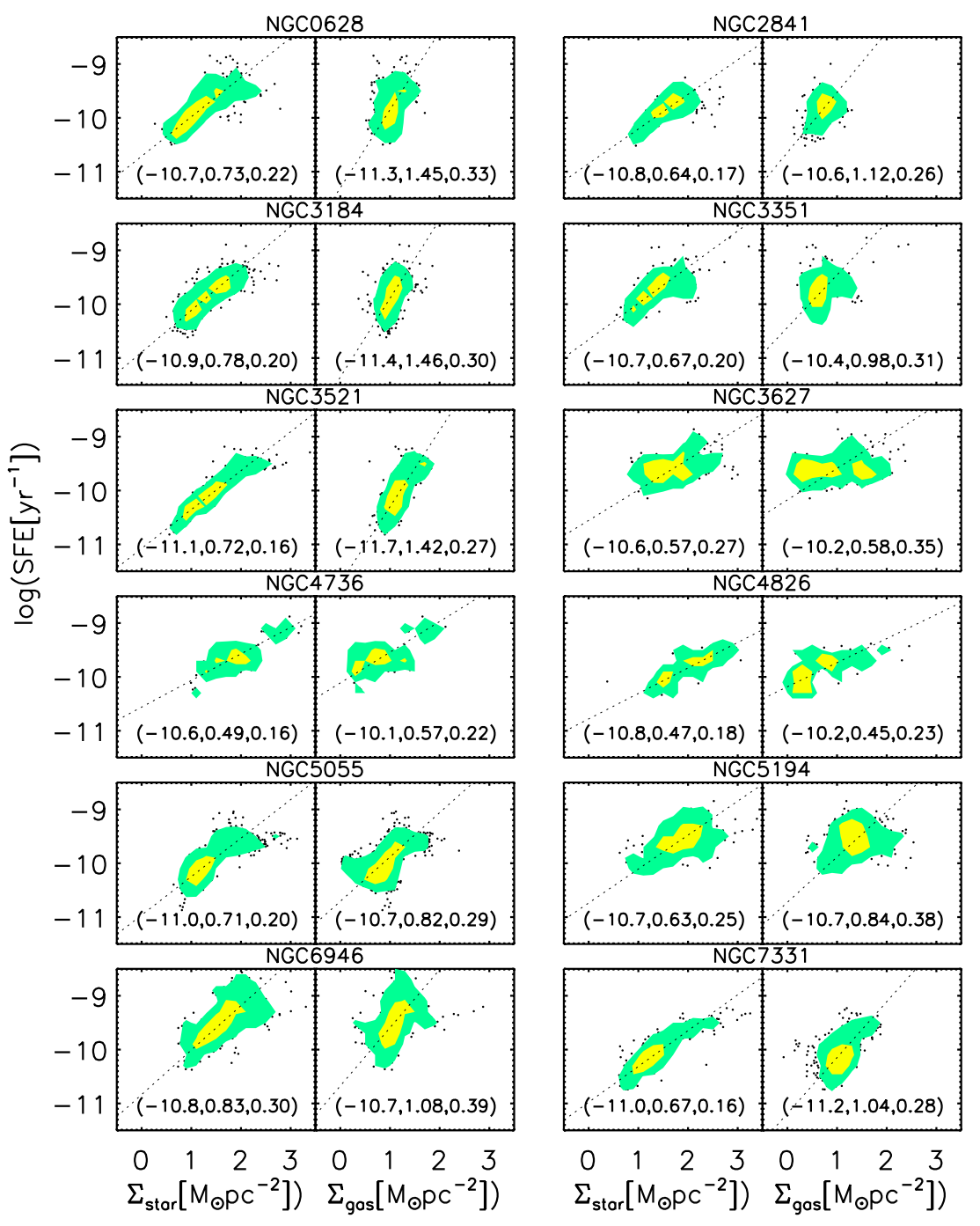

Figure 4. Extended Schmidt law at sub-kiloparsec resolution (the left side of each panel) compared to the KS law (the right side of each panel) in a sample of 12 spiral galaxies. The filled yellow and green areas enclose $50 \%$ and $90 \%$ of the total data points, respectively, while the dots are those outside the $90 \%$ area. The numbers listed in the parenthesize are in a sequence of interception, slope, and $1 \sigma$ observed scatter as given by the ordinary least-squares bisector fit (Isobe et al. 1990), while the dotted line is the best fit.

(A color version of this figure is available in the online journal.)

(2010) and Daddi et al. (2010). For the extended Schmidt law (Figure 3(c)), merging galaxies have offsets of 0.58 and 0.74 dex from the best fit to all objects and star-forming galaxies, respectively, which is also comparable to the case of the SFE$t_{\text {dyn }}$ relationships (0.5-0.7 dex by Genzel et al. 2010 and about 0.3 dex by Daddi et al. 2010). Thus even in the case of different CO-to- $\mathrm{H}_{2}$ factors for mergers, the basic idea that the SFE is regulated by the stellar density still holds.

\subsection{The Extended Schmidt Law at Sub-kiloparsec Resolution}

The result of the extended Schmidt law at sub-kiloparsec resolution in 12 spiral galaxies is shown in the left hand of each panel in Figure 4. It clearly indicates that the SFE is also a function of the stellar mass density for sub-kiloparsec regions in individual spiral galaxies. This demonstrates that the extended Schmidt law proposed for global galaxies in the above section also works at sub-kiloparsec resolution. Compared to the KS law as shown in the right hand of each panel, the extended Schmidt law shows significantly smaller scatter. Within individual galaxies, the observed scatter of the extended Schmidt law is on average 1.5 times smaller than that of the KS law. Across 12 galaxies, the mean and standard deviation of the slope of the extended Schmidt law is $0.66 \pm 0.11$ whose scatter is three times smaller than that of the KS law $(0.98 \pm 0.35)$.

Figure 5 shows the overall trend for all galaxies. A linear regression fit (IDL regress.pro) to gas mass, stellar mass, and SFR surface densities gives

$$
\Sigma_{\mathrm{SFR}} \propto \Sigma_{\text {gas }}^{0.80 \pm 0.01} \Sigma_{\text {star }}^{0.63 \pm 0.01}
$$

Again, the exponent of the stellar mass density is significantly from zero, indicating the importance of this quantity in predicting the SFR. The fit to SFE versus $\Sigma_{\text {star }}$ gives a slope of $0.75 \pm 0.01$ with the ordinary least-squares bisector method (Isobe et al. 1990). As shown in Figure 5, below SFE of $10^{-9.8} \mathrm{yr}^{-1}$ (horizontal dashed line), the slope becomes much steeper for the KS law, consistent with what found in Bigiel et al. (2008). On the other hand, such a large deviation is not seen for the extended Schmidt law. This further suggests the universality of the extended Schmidt law at sub-kiloparsec resolution.

Many previous spatially resolved studies of nearby galaxies have also noticed the trend of SFR as a function of stars. Ryder 


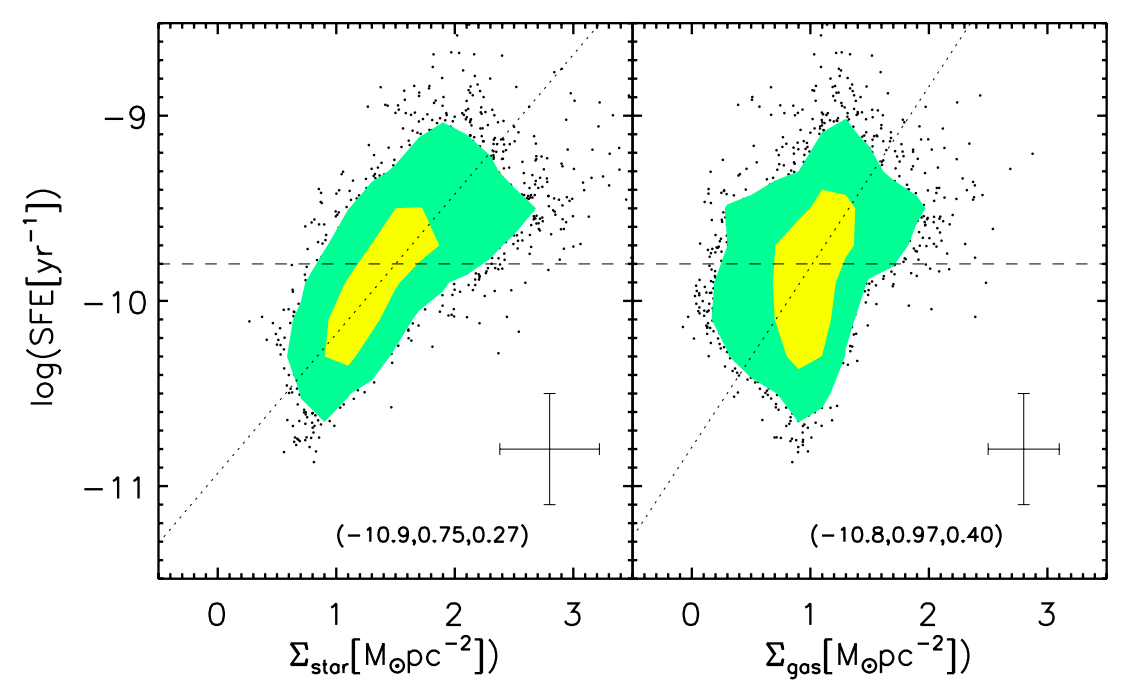

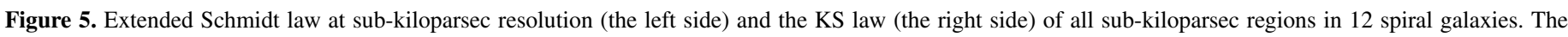

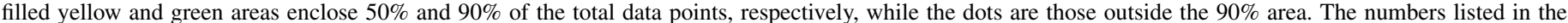

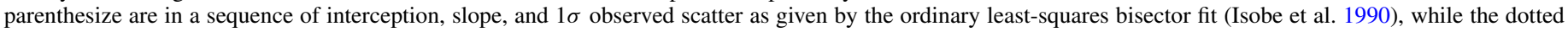
line is the best fit. The horizontal dashed line marks the transition below which the KS law has a much steeper slope (also see Bigiel et al. 2008).

(A color version of this figure is available in the online journal.)

\& Dopita (1994) found that the $\mathrm{H} \alpha$ emission spatially follows the distribution of $I$-band stellar emission in spiral disks. Their quantitative result gives $\Sigma_{\mathrm{H} \alpha} \propto \Sigma_{I \text { band }}^{0.64 \pm 0.37}$ within and among galaxies. In the LSB galaxies, Hunter et al. (1998) also found that the radial profile of the SFR follows that of the stellar mass density but not the gas density profile. Several other works have also noticed similar clues about the relationship between existing stars and star formation (Brosch et al. 1998; Hunter \& Elmegreen 2004). Recently, Leroy et al. (2008) have also pointed out a correlation between the SFE and stellar density but claimed different slopes (see their Figure 3): a slope of unity in the $\mathrm{H}$ I-dominated regime with $1<\log \left(\frac{\Sigma_{\text {star }}}{M_{\odot} \mathrm{pc}^{-2}}\right)<1.9$ and a constant trend (zero slope) in the $\mathrm{H}_{2}$-dominated regime of $\log \left(\frac{\Sigma_{\text {star }}}{M_{\odot} \mathrm{pc}^{-2}}\right)>1.9$. We re-analyzed their data and found two factors that cause this inconsistency. We note that the unity slope in that work is not from a fit and the overall variation in the SFE at $1<\log \left(\frac{\Sigma_{\text {star }}}{M_{\odot} \mathrm{pc}^{-2}}\right)<1.9$ is almost a factor of 10 , too large to constrain the slope. A direct fit to all of their data points gives a slope of 0.65 , close to our value. Also, they do not account for the color gradient in their stellar mass measurements, which would steepen the intrinsic slope. All of these works together strongly demonstrate the existence of the SFE as a function of the stellar mass at sub-kiloparsec resolution, while ours further indicate its significantly smaller scatter compared to the KS law.

\section{DISCUSSION}

\subsection{Test of Theoretical Models of the Star Formation Recipe}

\subsubsection{Is the Extended Schmidt Law Just Another Form of the KS Law?}

Can the extended Schmidt law be a result of the KS law and the relation between gas and stellar densities? Figure 6 shows the relation between $\Sigma_{\text {gas }}$ and $\Sigma_{\text {star }}$. Excluding the LSB and earlytype objects, the gas density scales with the stellar density with an observed scatter of 0.5 dex. If the extended Schmidt law is derived from the KS law and the $\Sigma_{\text {gas }}-\Sigma_{\text {star }}$, it should have a scatter of 0.7 dex given the scatter of the SFE $-\Sigma_{\text {gas }}$ relation of 0.5 dex, whereas the observed scatter of the extended Schmidt law is only 0.4 dex as listed in Table 4 . This suggests that the KS

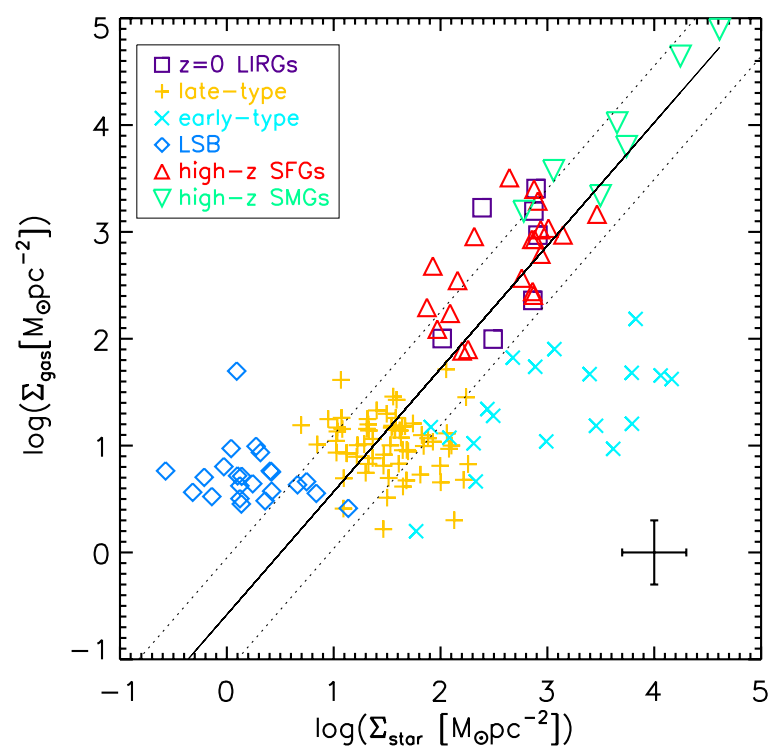

Figure 6. Gas mass surface density vs. stellar surface density. The solid line is the fit to galaxies excluding LSB and early-type ones. The dotted lines is the observed $1 \sigma$ scatter.

(A color version of this figure is available in the online journal.)

law and $\Sigma_{\text {gas }}-\Sigma_{\text {star }}$ relations are not fundamental relations that drive the SFE- $\Sigma_{\text {gas }}$ one. Stronger evidence comes from the LSB galaxies which do not follow either the KS law or $\Sigma_{\text {gas }}-\Sigma_{\text {star }}$ relation but do follow the SFE- $\Sigma_{\text {gas }}$ relation. In addition, the early-type galaxies also seem offset from the $\Sigma_{\text {gas }}-\Sigma_{\text {star }}$ relation but they do follow the extended Schmidt law. By invoking a different physical parameter $\left(\Sigma_{\text {star }}\right)$, the extended Schmidt law presents another star formation law that is not a simple recasting of the KS relation. In the remainder of this section, we will test several simple physical star formation models in order to understand its origin.

\subsubsection{Free Fall in a Star-dominated Potential}

While the KS law can be interpreted as a free fall in a gasdominated gravitational potential, we note that the extended Schmidt law also is consistent with the idea of free fall in a 
stellar potential. The SFR can be expressed as the amount of gas collapsing into stars within a given timescale:

$$
\Sigma_{\mathrm{SFR}}=\frac{\eta \Sigma_{\mathrm{gas}}}{\tau}
$$

where $\eta$ gives the fraction of the total gas that collapses into stars and $\tau$ describes the duration of gas collapse. A free-fall gas collapse in a star-dominated potential has a timescale:

$$
\begin{aligned}
\tau_{\mathrm{ff}} & =\frac{1}{4} \sqrt{\frac{3 \pi}{2 G\left(\rho_{\mathrm{gas}}+\rho_{\mathrm{star}}\right)}} \approx \frac{1}{4} \sqrt{\frac{3 \pi}{2 G \rho_{\mathrm{star}}}}=\frac{1}{4} \sqrt{\frac{3 \pi h_{\mathrm{star}}}{G \Sigma_{\mathrm{star}}}} \\
& =3.5 \times 10^{8} \mathrm{yr}\left(\frac{h_{\mathrm{star}}}{1 \mathrm{kpc}}\right)^{0.5}\left(\frac{1 M_{\odot} \mathrm{pc}^{-2}}{\Sigma_{\mathrm{star}}}\right)^{0.5},
\end{aligned}
$$

where $\rho_{\text {gas }}, \rho_{\text {star }}, \Sigma_{\text {star }}$, and $h_{\text {star }}$ are the gas mass volume density, stellar mass volume density, stellar mass surface density, and the stellar scale height, respectively. For a self-gravitating stellar disk, $\Sigma_{\text {star }}=2 \rho_{\text {star }} h_{\text {star }}$. This simple interpretation predicts the observed power index of 0.5 for $\Sigma_{\text {star }}$, if $\rho_{\text {star }} \gg \rho_{\text {gas }}$ and $h_{\text {star }}=$ constant. The comparison of Equation (8) to the observed relation gives

$$
\eta \approx 2 \% \text {. }
$$

How well does this scenario represent reality? How does the stellar potential act on the gas collapse? As shown above, the above derivation assumes two conditions $h_{\text {star }}=$ constant and $\rho_{\text {star }} \gg \rho_{\text {gas }}$. While there is evidence that the stellar scale height remains constant with radius within a galaxy (van der Kruit \& Searle 1981; Fry et al. 1999), the variation among galaxies may contribute to the scatter of the correlation under this interpretation. The condition of $\rho_{\text {star }} \gg \rho_{\text {gas }}$ is true for general high-surface-brightness galaxies but not for LSB ones, as shown in Figure 6 which shows that the gas potential dominates over the stellar one. If the gas density is included in the correlation as $\mathrm{SFE} \propto\left(\Sigma_{\text {gas }}+\Sigma_{\text {star }}\right)^{0.5}$, the correlation will not improve, but instead LSB galaxies will be offset toward the high density end. There is no room for additional gas self-gravity in the empirical relation.

\subsubsection{Pressure-regulated $\mathrm{H}_{2}$ Formation}

The prerequisite to star formation is formation of cold molecular $\mathrm{H}_{2}$. Many works have highlighted the role of the stellar gravity in regulating $\mathrm{H}_{2}$ formation (Elmegreen 1993; Wong \& Blitz 2002; Blitz \& Rosolowsky 2004, 2006). We explore here whether the extended Schmidt law actually reflects the process of $\mathrm{H}_{2}$ production from $\mathrm{H}$. Quantitatively, the $\mathrm{H}_{2}$ to-H I mass ratio $\left(R_{\mathrm{mol}}\right)$ can be written as a function of pressure with a power index of $\gamma$ :

$$
R_{\mathrm{mol}}=\mathrm{H}_{2} / \mathrm{H} \mathrm{I} \propto P_{\mathrm{ext}}^{\gamma} .
$$

Blitz \& Rosolowsky (2004) estimate the external pressure $P_{\text {ext }}$ as the mid-plane pressure in an infinite two-fluid isothermal disk where the gas scale height is much less than the stellar height:

$$
\begin{aligned}
P_{\text {ext }}= & (2 G)^{0.5} \Sigma_{\text {gas }} v_{\text {gas }}\left[\rho_{\text {star }}^{0.5}+\left(\frac{\pi}{4} \rho_{\text {gas }}\right)^{0.5}\right] \\
= & 0.84\left(G \Sigma_{\text {star }}\right)^{0.5} \Sigma_{\text {gas }} \frac{v_{\text {gas }}}{h_{\text {star }}^{0.5}} \\
= & \left(272 \mathrm{~cm}^{-3} \mathrm{~K}\right)\left(\frac{\Sigma_{\text {gas }}}{M_{\odot} \mathrm{pc}^{-2}}\right)\left(\frac{\Sigma_{\text {star }}}{M_{\odot} \mathrm{pc}^{-2}}\right)^{0.5} \\
& \times\left(\frac{v_{\text {gas }}}{\mathrm{km} \mathrm{s}^{-1}}\right)\left(\frac{h_{\text {star }}}{\mathrm{pc}}\right)^{-0.5}(k),
\end{aligned}
$$

where $\Sigma_{\text {gas }}$ is the mid-plane gas surface density, $v_{\text {gas }}$ is the vertical velocity dispersion of the gas disk, $\rho_{\text {star }}$ is the mid-plane stellar volume density, $\rho_{\text {gas }}$ is the mid-plane gas volume density, $\Sigma_{\text {star }}$ is the mid-plane stellar surface density, $h_{\text {star }}$ is the stellar scale height, and $k$ is the Boltzmann constant. By assuming constant $v_{\text {gas }}$ for the gas disk and constant $h_{\text {star }}$ for the stellar disk, Equation (11) has been demonstrated observationally with $\gamma$ around 1.0 (Blitz \& Rosolowsky 2006). Under this assumption and that stars form from molecular gas, the star formation prescription can be written as

$\Sigma_{\mathrm{SFR}}=\frac{\eta_{\mathrm{H}_{2}} \Sigma_{\mathrm{H}_{2}}}{\tau}=\frac{\eta_{\mathrm{H}_{2}}}{\tau} \frac{R_{\mathrm{mol}}}{1+R_{\mathrm{mol}}} \Sigma_{\mathrm{gas}}=\frac{\eta_{\mathrm{H}_{2}}}{\tau} \frac{\left(P_{\mathrm{ext}} / P_{0}\right)^{\gamma}}{1+\left(P_{\mathrm{ext}} / P_{0}\right)^{\gamma}} \Sigma_{\mathrm{gas}}$,

where $\eta_{\mathrm{H}_{2}}$ is the fraction of the molecular gas that ends up in stars, $\tau$ is the timescale for the collapse of molecular clouds to stars, $\gamma=0.92$, and $P_{0} / k$ is $4.3 \times 10^{4} \mathrm{~cm}^{-3} \mathrm{~K}$ as given by observation in Blitz \& Rosolowsky (2006). The $\frac{\eta_{\mathrm{H}_{2}}}{\tau}$ is observed to be a constant (e.g., Gao \& Solomon 2004; Wu et al. 2005; Leroy et al. 2008; Genzel et al. 2010). We now discuss $\Sigma_{\text {SFR }}$ in two extreme pressure regimes.

1. H I-dominated galaxies ( $\left.P_{\text {ext }} / P_{0} \ll 1, R_{\text {mol }} \ll 1\right)$ : the above equation gives

$$
\Sigma_{\mathrm{SFR}} \propto\left(P_{\mathrm{ext}} / P_{0}\right)^{\gamma} \Sigma_{\mathrm{gas}} \propto \Sigma_{\mathrm{star}}^{0.5 \gamma} \Sigma_{\mathrm{gas}}^{1.0+\gamma}
$$

for constant $h_{\text {star }}$ and $v_{\text {gas }}$. At $\gamma=0.92$, the above equation predicts roughly the same power index for the $\Sigma_{\text {star }}$ as we observe but almost two times larger for that of $\Sigma_{\text {gas }}$.

2. $\mathrm{H}_{2}$-dominated galaxies $\left(P_{\text {ext }} / P_{0} \gg 1, R_{\text {mol }} \gg 1\right)$ : it is obvious in this regime there is no dependence of $\Sigma_{\mathrm{SFR}}$ on $\Sigma_{\text {star }}$, inconsistent with the extended Schmidt law for $\mathrm{H}_{2}$ dominated circumnuclear star-forming regions and LIRGs.

\subsubsection{Pressure-supported Star Formation}

The scenario of pressure-supported star formation assumes that star formation is regulated by the pressure balance between gas collapse and feedback from stars (Thompson et al. 2005), i.e., weak stellar feedback leaves the gas collapse unimpeded, resulting in enhanced star formation, which in turn increases the feedback to prevent the further gas collapse; strong feedback prevents the efficient gas collapse, which lowers the amount of newly formed stars and thus decreases the feedback strength. Quantitatively, we have

$$
P_{\mathrm{ext}}=P_{\mathrm{SFR}}
$$

Thompson et al. (2005) estimate the total pressure from star formation as a sum of the supernovae feedback and radiation pressure:

$$
\begin{aligned}
P_{\mathrm{SFR}} & \approx\left(P_{\mathrm{SN}}+P_{\mathrm{RP}}\right) \approx\left(5 n_{1}^{-1 / 4} E_{51}^{13 / 14}+1\right) P_{\mathrm{RP}} \\
& \approx\left(5 n_{1}^{-1 / 4} E_{51}^{13 / 14}+1\right) \epsilon c \Sigma_{\mathrm{SFR}},
\end{aligned}
$$

where $P_{\mathrm{SN}}$ is the pressure from supernova, $P_{\mathrm{RP}}$ is the radiation pressure from massive stars, $n_{1}$ is the density of the ISM in the unit of $1 \mathrm{~cm}^{-3}, E_{51}$ is the supernova energy in units of $10^{51} \mathrm{erg}, \epsilon$ is the conversion efficiency from the stellar mass into radiation $\left(\epsilon \sim 10^{-3}\right.$ for a Salpeter IMF), and $c$ is the speed of light. In general quiescent galaxies, the ISM density is low and the pressure is dominated by supernova, while in LIRGs the radiation pressure starts to become important or even dominates. 
Using the Equation (12) for $P_{\text {ext }}$, we have

$$
\begin{aligned}
\frac{\Sigma_{\mathrm{SFR}}}{M_{\odot} \mathrm{yr}^{-1} \mathrm{pc}^{-2}}= & \frac{1.9 \times 10^{-10}}{\left(5 n_{1}^{-1 / 4} E_{51}^{13 / 14}+1\right)}\left(\frac{\Sigma_{\mathrm{gas}}}{M_{\odot} \mathrm{pc}^{-2}}\right) \\
& \times\left(\frac{\Sigma_{\mathrm{star}}}{M_{\odot} \mathrm{pc}^{-2}}\right)^{0.5}\left(\frac{v_{\mathrm{gas}}}{\mathrm{km} \mathrm{s}^{-1}}\right)\left(\frac{h_{\mathrm{star}}}{\mathrm{pc}}\right)^{-0.5} .
\end{aligned}
$$

Comparing to the observed correlation (Equation (22)), the above equation produces not only the exact power indices for both $\Sigma_{\text {star }}$ and $\Sigma_{\text {gas }}$ but also a similar constant. The caveat to this explanation is again offered by the LSB galaxies where the gas gravity cannot be neglected compared to the stellar term as shown in Figure 6 and as discussed above.

\subsubsection{Summary: Causal or Casual?}

We compared the extended Schmidt law to some physical star formation models including gas free fall in the stellar potential, pressure-regulated $\mathrm{H}_{2}$ formation, and pressure-supported star formation. All of the models invoke roles of the existing stars in star formation through stellar gravity on gas, and the first and third ones predict not only the same power indices but also similar normalizations to the data. However, the assumption that stars dominate the mass seems unreasonable for the LSB galaxies, at least in the case of hydrostatic equilibrium. On the other hand, the stellar gravity can affect gas motion critically in configurations where stars show spatial and velocity differences from gas, such as stellar bars. An example of this may be seen in the numerical simulation of gas-dominated merging galaxies (Hopkins et al. 2009a, 2009b). Springel \& Hernquist (2005) and Robertson et al. (2006) have shown that gas-rich mergers result in disk galaxies instead of elliptical galaxies. The reason for this is not just that gas-rich mergers have too much gas to consume but also that they lack existing stars. Stars are collisionless and can relax their orbits violently during merging. Gas, on the other hand, is collisional and cannot relax rapidly, requiring angular momentum to be removed in order to form stars (Hopkins et al. $2009 b$ ). With non-axisymmetric distortion as induced by the secondary galaxy, the gravity of these stars thus provides the most efficient way to remove the gaseous angular momentum. Its efficiency far exceeds those of shock compression, gravity of the secondary galaxy, and self-gravity of the gas itself. Hopkins et al. (2009a) have derived an analytic expression that captures the role of existing stars: $f_{\text {starburst }} / f_{\text {gas }} \propto f_{\text {star }}$, where $f_{\text {starburst }}$ is the fraction of the total mass that forms stars, $f_{\text {gas }}$ is initial gas mass fraction, and $f_{\text {star }}$ is initial stellar mass fraction. Determining whether a similarly critical influence of stars on gas flow and processing is also obtained in LSB and normal galaxies will probably require extensive simulations.

Since the physical models of star formation do not translate directly into the extended Schmidt law, one is not free to interpret the latter as a causal formula implying that stellar gravity regulates SFE. Instead, $\Sigma_{\text {star }}$ may be a proxy for other physical parameters or a combination thereof, signifying regulation by more subtle or complex physics. $\Sigma_{\text {star }}$ may represent the total kinetic and/or radiation energy dumped into the ISM by stars or the total metal enrichment over the galaxy's history. For example, the metal abundance is critical in ISM cooling and formation of dust grains on which $\mathrm{H}_{2}$ can form efficiently. Theoretical models do confirm the significant deviation of the KS law in the low-metallicity environment (Krumholz et al. 2009; Gnedin \& Kravtsov 2010; Papadopoulos \& Pelupessy 2010).
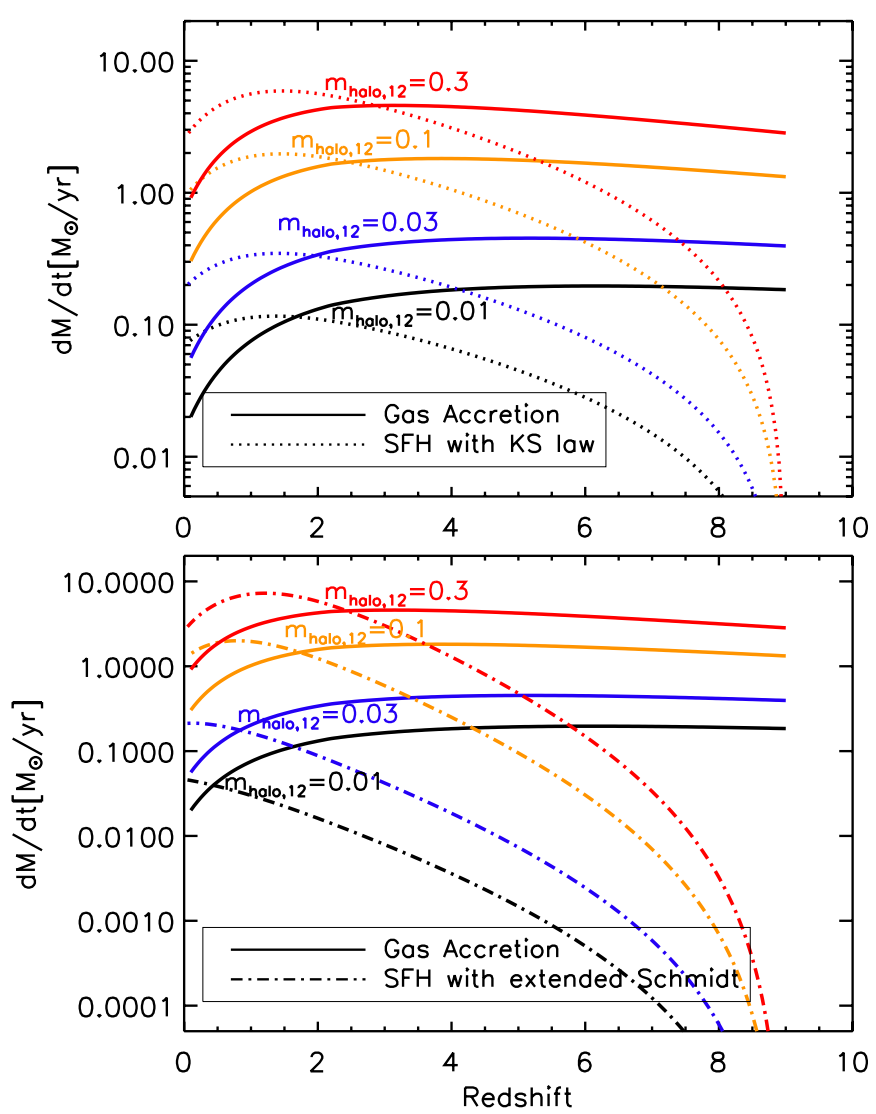

Figure 7. Upper: the evolution of $\operatorname{SFR}(z)$ (dotted lines) as predicted by the KS law for given gas accretion histories (solid lines with the same colors) in four different halo masses where $m_{\text {halo, } 12}$ is the halo mass at $z=0$ in units of $10^{12} M_{\odot}$. Lower: the SFR histories with the extended Schmidt law.

(A color version of this figure is available in the online journal.)

\subsection{Implications for the Star-forming Main Sequence}

We now discuss the implication of the extended Schmidt law for galaxy formation and evolution with focus on the main sequence of star-forming galaxies. Studies have shown that stars form mainly in the blue star-forming galaxies while there is little star formation in red galaxies. Such a bi-modality has been well established through various observations (e.g., Blanton et al. 2003). While red galaxies show a relation between the UV/ optical color and luminosity, the SFRs of blue galaxies are found to correlate with stellar masses, a relationship with a slope a bit below unity and a small dispersion of $\lesssim 0.3$ dex (Brinchmann et al. 2004; Elbaz et al. 2007; Daddi et al. 2007; Zheng et al. 2007; Noeske et al. 2007a; Chen et al. 2009; Oliver et al. 2010; Rodighiero et al. 2010). This so-called main sequence of starforming galaxies and its evolution have provided important constraints on the mechanism driving the rapid evolution of the cosmic SFR density. For example, an evolving stellar IMF is able to explain the evolution of the main sequence as proposed by Davé (2008). A more general interpretation would attempt to quantify the star formation history ( $\mathrm{SFH}$ ), since

$$
\begin{aligned}
& M_{\text {star }}\left(z_{0}\right) \propto \int_{z_{\text {form }}}^{z_{0}} \operatorname{SFR}(z) d z=\operatorname{SFR}\left(z_{0}\right) \int_{z_{\text {form }}}^{z_{0}} \frac{\operatorname{SFR}(z)}{\operatorname{SFR}\left(z_{0}\right)} d z \\
& \quad=\operatorname{SFR}\left(z_{0}\right) \int_{z_{\text {form }}}^{z_{0}} \operatorname{SFH}(z) d z
\end{aligned}
$$

where $z_{0}$ is the observed redshift of a galaxy, $z_{\text {form }}$ is the redshift where the galaxy starts to form, and SFH is the star 

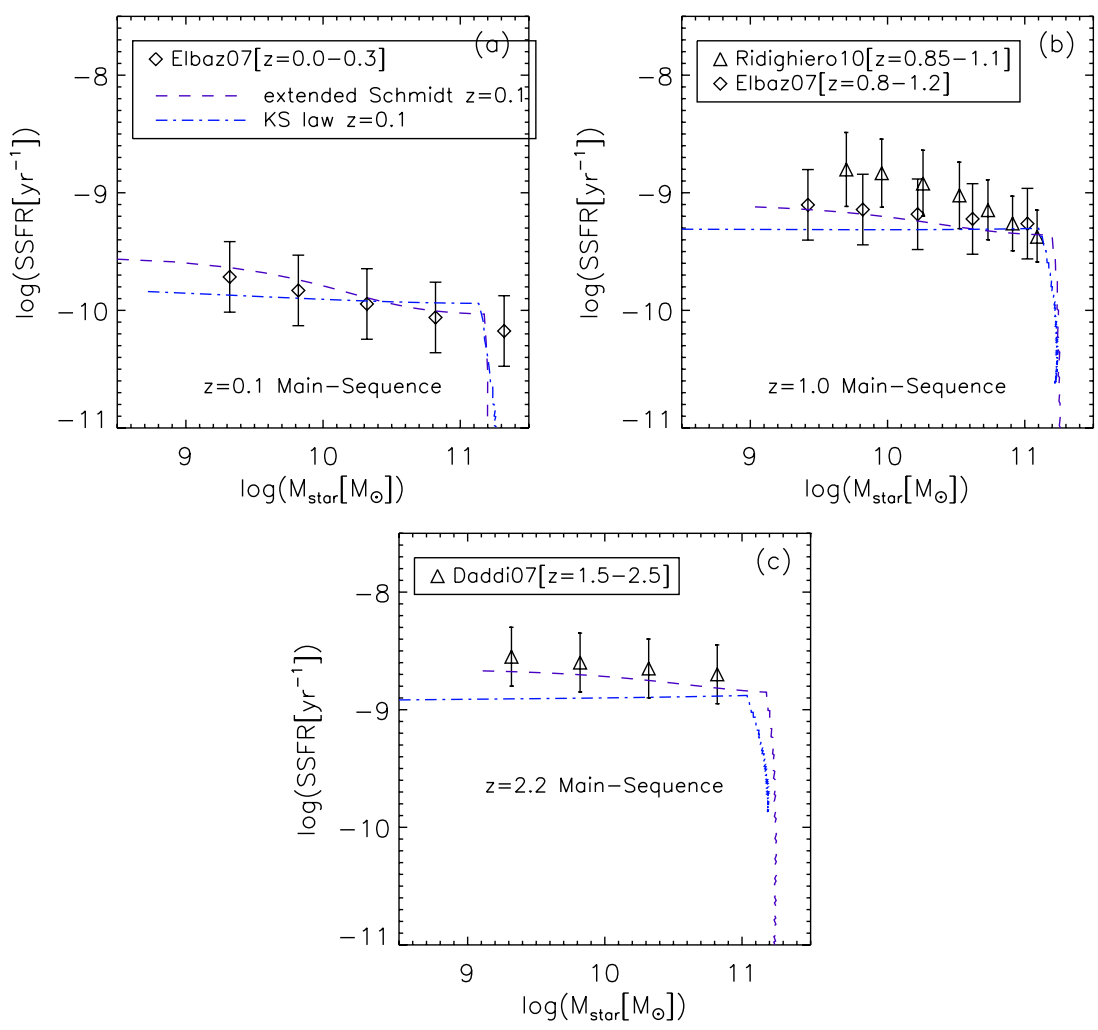

Figure 8. Observed star-forming main sequence from $z=0$ to $z=2$ compared to the predictions of implementing either the extended Schmidt law (dashed lines) or the KS law (dot-dashed lines) into the analytic model of gas accretion in the $\Lambda \mathrm{CDM}$ cosmology.

(A color version of this figure is available in the online journal.)

formation history normalized by the current SFR. Current numerical simulations and analytic models have difficulties in producing the observed slope below unity without invoking ad hoc mechanisms to delay star formation in low mass systems (Noeske et al. 2007b; Davé 2008; Bouché et al. 2010). This can be seen from Equation (18). If all galaxies have a similar smooth shape of SFH and form at the same redshift, then Equation (18) gives a slope of unity. To have a shallower slope, the integral of the SFH normalized by the current SFR needs to be smaller for a lower mass galaxy (see Equation (18)), for example, $z_{\text {form }}$ can be lower for a lower mass galaxy. This can be also rephrased as shorter characteristic star-formation timescale or late onset of star formation in a lower mass galaxy. However, in numerical simulations and semi-analytic models, the gas accretion is determined by the well-known dark matter halo growth in $\Lambda$ CDM cosmology. Thus, SFH is not a free parameter to adjust (Davé 2008). The growth of the dark matter halo follows $\dot{M}_{\text {halo }} \propto M_{\text {halo }}^{s}$ with $s$ above unity (e.g., Neistein \& Dekel 2008), which would result in a slope of the main sequence above unity if the SFR follows that of the dark matter halo growth. In current models, ad hoc mechanisms are thus proposed to delay star formation in low-mass galaxies, such as a very strong feedback or a mass floor below which the gas cannot be accreted (e.g., Davé 2008; Bouché et al. 2010). Compared to the KS law that is now widely invoked in numerical simulations and semi-analytic models, the extended Schmidt law indicates a slow SFR increase at early times due to the lack of existing stars and fast evolution at late times for a given gas accretion history. This naturally introduces a delayed onset of star formation in a low-mass galaxy, which is the key to explaining the star-forming main sequence with a slope below unity. In the following, we show quantitatively that the delayed star formation in a low mass system is a natural result of star formation that is governed by the extended Schmidt law.

We follow exactly Bouché et al. (2010) to construct the growth of the dark matter halo and gas accretion. For a given gas accretion history, star formation occurs following either the extended Schmidt law or the KS law. To apply these two laws, we assume the evolution of the half-light radius $\left(R_{1 / 2}\right)$ from Papovich et al. (2011). The numerical calculations include four elements:

1. The dark matter halo growth rate follows:

$$
\dot{M}_{\text {halo }}=510 M_{\text {halo, } 12}^{s}((1+z) / 3.2)^{t} M_{\odot} \mathrm{yr}^{-1},
$$

where $M_{\text {halo, } 12}=M_{\text {halo }} / 10^{12} M_{\odot}, s=1.1$, and $t=2.2$.

2. The gas accretion rate is given by

$$
\dot{M}_{\text {gas,in }}=\epsilon_{\text {in }} f_{\mathrm{b}} \dot{M}_{\text {halo }} .
$$

Similar to Bouché et al. (2010), $f_{\mathrm{b}}$ is the baryonic fraction of $0.18 . \epsilon_{\text {in }}$ is the accretion efficiency that is equal to 0.7 at $z>2.2$ where cold accretion mode dominates. Due to accumulation of stars and hot gas, the accretion efficiency must decrease with time. For $z<2.2$, Bouché et al. (2010) simply assumed $\epsilon_{\text {in }}=f(z) \times 0.7$ where $f(z)$ is a linear function of time with $f(2.2)=1$ and $f(0)=0.5$. For $M_{\text {halo }}$ above $10^{12} M_{\odot}$ where the cold mode accretion is not important, $\epsilon_{\text {in }}=0$ as discussed by Bouché et al. (2010). However, we do not introduce a low-mass floor $\left(M_{\text {halo }}=\right.$ $10^{11} M_{\odot}$ ) below which $\epsilon_{\text {in }}=0$, which is used to fit the observed main sequence as shown by Bouché et al. (2010). This is the main difference of our model besides adopting the extended Schmidt law. 
3. At each redshift, the net gas accretion is given by

$$
\dot{M}_{\mathrm{gas}}=\dot{M}_{\mathrm{gas}, \text { in }}-(1-R) \times \mathrm{SFR}-\dot{M}_{\mathrm{gas}, \text { out }},
$$

where $R$ is the recycled gas fraction and equal to 0.52 for a Chabrier IMF in this study. $\dot{M}_{\text {gas,out }}=a \times$ SFR is the gas outflow where $a$ is set to be zero by assuming the outflow eventually falls back to form stars. Unlike the above two (Equations (19) and (20)) that are solely determined by the dark matter growth, this equation will produce different results for different star formation laws.

4. Star formation follows either the extended Schmidt law:

$$
\frac{\mathrm{SFE}}{\mathrm{yr}^{-1}}=10^{-10.28}\left(\frac{\Sigma_{\text {star }}}{M_{\odot} \mathrm{pc}^{-2}}\right)^{0.48}
$$

or the KS law (using the exponent from the fit in this study):

$$
\frac{\Sigma_{\mathrm{SFR}}}{M_{\odot} \mathrm{yr}^{-1} \mathrm{pc}^{-2}}=10^{-9.90}\left(\frac{\Sigma_{\mathrm{gas}}}{M_{\odot} \mathrm{pc}^{-2}}\right)^{1.38}
$$

where $\Sigma=\frac{0.5 M}{\pi R_{1 / 2}^{2}}$. Following Papovich et al. (2011), the half-light radius $R_{1 / 2}(z)$ is given by

$$
\frac{R_{1 / 2}}{\mathrm{kpc}}=1.7 \frac{H(z=4)}{H(z)},
$$

where $H(z=4)=430 \mathrm{~km} \mathrm{~s}^{-1} \mathrm{Mpc}^{-1}$.

The above six equations are solved numerically with the formation $z_{\text {form }}=9$, where the initial gas and stellar mass are set to zero, and a series of initial dark matter masses are assumed. Examples of gas accretion and SFR histories are shown in Figure 7 for both KS and extended Schmidt laws. With the KS law, star formation responds only to the accumulated gas and thus quickly reaches the state where the SFR follows more or less the gas accretion history at later times (e.g., Papovich et al. 2011). As shown in Figure 7, the SFHs of different mass systems are quite similar at $z<7$ with similar peak redshift and slopes on both sides of the peak. This implies a unit slope for the star-forming main sequence as indicated by Equation (18). On the other hand, the SFH given by the extended Schmidt law slowly increases at early time and increases more slowly for lower mass systems simply as a result of smaller existing stellar populations. The SFHs peak at lower redshift for lower mass systems as shown in Figure 7, which naturally introduces a delay mechanism that is required to explain the main sequence. The quantitative comparisons to the observed main sequences in term of SSFR (specific star formation rate) versus $M_{\text {star }}$ are shown in Figure 8. While both relations produce more or less the correct normalizations, the KS law never produces a negative slope, while the prediction by the extended Schmidt law is consistent with the observed data from $z=0$ all the way up to $z=2$. Such a consistency is reached without introducing ad hoc mechanisms to delay star formation in low mass systems, unlike other studies based on the KS law (Noeske et al. 2007b; Davé 2008; Bouché et al. 2010). Figure 9 collects current studies of the SSFR evolution including both normalization at $\log M_{\text {star }} / M_{\odot}=$ 10 and the slope. In general, these two relations (extended Schmidt and KS laws), as well as the numerical simulations, all predict the rapid evolution of the SSFR, which reflects the gas accretion history. Among them, however, the extended Schmidt law produces the best match to the observed data, although a discrepancy still exists at high- $z(z>3)$. As shown in the
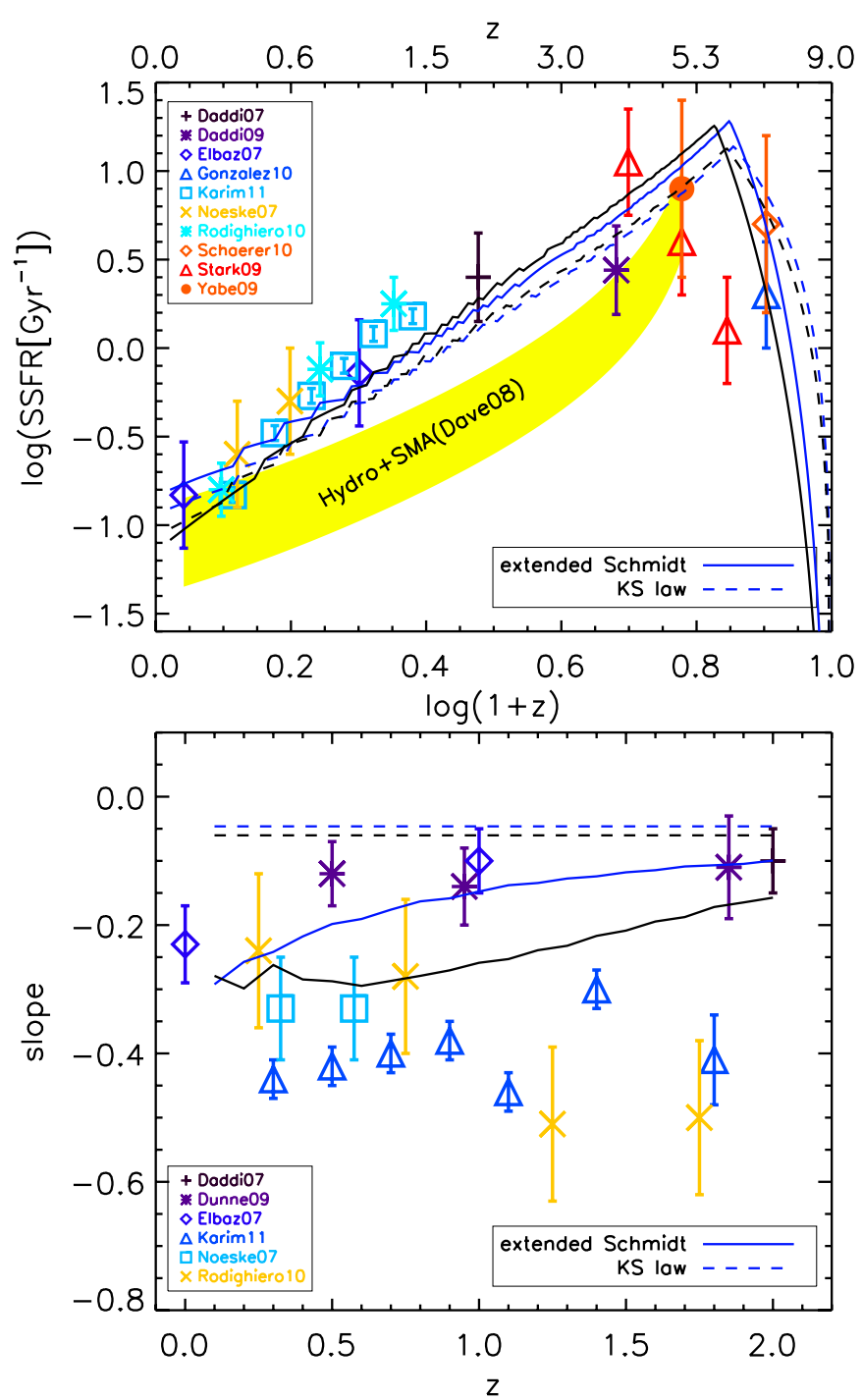

Figure 9. Upper panel: the observed evolution of the specific star formation rate (SSFR) for $M_{\text {star }}=10^{10} M_{\odot}$ compared to the predictions by the extended Schmidt (solid lines) and KS laws (dashed lines). The data are taken directly from the literature except for Stark et al. (2009) where we further correct the extinction according to the luminosity- and redshift-dependent extinction correction curve of Bouwens et al. (2009). "SMA" stands for the semi-analytic model. Lower panel: the evolution of the observed slope (specific SFR $\propto M_{\text {star }}^{\text {slope }}$ ) as a function of the redshift from different studies. The solid and dashed lines show the result for the extended and KS laws, respectively, while the blue and black colors indicate the galaxy size evolution of $\propto(H(z=4) / H(z))$ and $\propto$ $(H(z=4) / H(z))^{0.4}$, respectively. The data points correspond to the following references described in the legend: Daddi et al. 2007, 2009; Elbaz et al. 2007; González et al. 2010; Karim et al. 2011; Noeske et al. 2007a; Rodighiero et al. 2010; Schaerer \& de Barros 2010; Stark et al. 2009; Yabe et al. 2009; and Dunne et al. 2009.

(A color version of this figure is available in the online journal.)

lower panel of Figure 9, although different slopes of the main sequence have been observed by different studies, all of them are negative. It is clearly shown that the extended Schmidt law produces much more consistent values with the observed data compared with the KS law. The above result about the predicted slope by the KS law depends little on the numerical values of the physical parameters invoked in Equations (19)-(24), except for the $s$ parameter that is unlikely to be below unity. A steeper slope by the extended Schmidt law can be produced if the galaxy size evolves slower $\left(\propto(H(z=4) / H(z))^{0.4}\right)$ shown as black lines in the figure. 
Recently, Dutton et al. (2010) have reproduced the observed main sequence through the semi-analytical model with the star formation recipe of the pressure-regulated $\mathrm{H}_{2}$ formation. The resulting two-power-law star formation relation has the same slope as the KS one at the high density regime but a much steeper slope (2.84) at the low density end. This further indicates that the pure KS law overpredicts the SFR at the early stage of galaxy evolution. Without imposing ad hoc mechanisms to delay star formation in a low mass system, the extended Schmidt law does provide a new way to understand the star-forming main sequence and its evolution.

\section{CONCLUSIONS}

1. We demonstrate empirically the existence of a tight correlation between the star formation efficiency $\left(\mathrm{SFE}=\Sigma_{\mathrm{SFR}} /\right.$ $\left.\Sigma_{\text {gas }}\right)$ and the stellar mass density $\left(\Sigma_{\text {star }}\right)$, referred as the extended Schmidt law. The correlation was derived by looking for the dependence of $\Sigma_{\mathrm{SFR}}$ on $\Sigma_{\text {gas }}$ and $\Sigma_{\text {star }}$. It has a power index of $0.48 \pm 0.04$ and holds over five orders of magnitude in the stellar mass density for various types of galaxies including the LSB ones that deviate significantly from the KS law.

2. We further show that the extended Schmidt law also applies to spatially resolved regions at sub-kiloparsec resolution. In a sample of 12 spiral galaxies, the extended Schmidt law not only holds for LSB regions but also shows significantly smaller scatters, a factor of 1.5 and 3 smaller within and across galaxies, respectively, compared to the KS law.

3. The extended Schmidt law may suggest a critical role for existing stellar populations in ongoing star formation activity. Alternatively, it may be a manifestation of more complex physics where $\Sigma_{\text {star }}$ is a proxy for other variables or processes. The comparison of the extended Schmidt law to several simple physical models indicates that models of gas free fall in stellar gravitational potential and pressuresupported star formation produce not only the same power index but also a similar normalization. However, this success is limited to some cases, and the exact physical interpretation of the extended Schmidt law needs further exploration.

4. By applying this extended Schmidt law to an analytic model of gas accretion in $\Lambda C D M$ cosmology, the observed main sequence of star-forming galaxies is well reproduced in the model without the need for ad hoc mechanisms to delay star formation in low mass systems.

We thank the anonymous referee for the detailed and constructive comments. We also thank Daniel Dale, Leslie K. Hunt, Eva Schinnerer, and Bruce G. Elmegreen for careful reading and comments. The work is supported through the Spitzer 5MUSES Legacy Program 40539. The authors acknowledge support by NASA through awards issued by JPL/Caltech. This work was based on observations made with the Spitzer Space Telescope, which is operated by JPL/Caltech under a contract with NASA. This research has made use of the NASA/IPAC Extragalactic Database (NED) which is operated by the Jet Propulsion Laboratory, California Institute of Technology, under contract with the National Aeronautics and Space Administration. Funding for the SDSS and SDSS-II has been provided by the Alfred P. Sloan Foundation, the Participating Institutions, the National Science Foundation, the U.S. Department of Energy, the National Aeronautics and Space Administration, the Japanese
Monbukagakusho, the Max Planck Society, and the Higher Education Funding Council for England. The SDSS Web site is http://www.sdss.org/.

\section{REFERENCES}

Bigiel, F., Leroy, A., Walter, F., Brinks, E., de Blok, W. J. G., Madore, B., \& Thornley, M. D. 2008, AJ, 136, 2846

Blanton, M. R., et al. 2003, ApJ, 594, 186

Blitz, L., \& Rosolowsky, E. 2004, ApJ, 612, L29

Blitz, L., \& Rosolowsky, E. 2006, ApJ, 650, 933

Boissier, S., Prantzos, N., Boselli, A., \& Gavazzi, G. 2003, MNRAS, 346, 1215 Bouché, N., et al. 2010, ApJ, 718, 1001

Bouwens, R. J., et al. 2009, ApJ, 705, 936

Brinchmann, J., Charlot, S., White, S. D. M., Tremonti, C., Kauffmann, G., Heckman, T., \& Brinkmann, J. 2004, MNRAS, 351, 1151

Brosch, N., Heller, A., \& Almoznino, E. 1998, ApJ, 504, 720

Bruzual, A. G. 2007, arXiv:astro-ph/0703052

Bruzual, G., \& Charlot, S. 2003, MNRAS, 344, 1000

Bryant, P. M., \& Scoville, N. Z. 1999, AJ, 117, 2632

Chabrier, G. 2003, PASP, 115, 763

Chen, Y.-M., Wild, V., Kauffmann, G., Blaizot, J., Davis, M., Noeske, K., Wang, J.-M., \& Willmer, C. 2009, MNRAS, 393, 406

Courteau, S. 1996, ApJS, 103, 363

Cox, A. L., Sparke, L. S., Watson, A. M., \& van Moorsel, G. 2001, AJ, 121, 692 Crocker, A. F., Bureau, M., Young, L. M., \& Combes, F. 2011, MNRAS, 410, 1197

Crosthwaite, L. P., \& Turner, J. L. 2007, AJ, 134, 1827

Daddi, E., et al. 2007, ApJ, 670, 156

Daddi, E., et al. 2009, ApJ, 694, 1517

Daddi, E., et al. 2010, ApJ, 713, 686

Davé, R. 2008, MNRAS, 385, 147

de Vaucouleurs, G., de Vaucouleurs, A., \& Corwin, H. G. 1976, University of

Texas Monographs in Astronomy (Austin, TX: Univ. of Texas Press)

Dickman, R. L., Snell, R. L., \& Schloerb, F. P. 1986, ApJ, 309, 326

Downes, D., \& Solomon, P. M. 1998, ApJ, 507, 615

Dunne, L., et al. 2009, MNRAS, 394, 3

Dutton, A. A., van den Bosch, F. C., \& Dekel, A. 2010, MNRAS, 405, 1690

Elbaz, D., et al. 2007, A\&A, 468, 33

Elmegreen, B. G. 1993, ApJ, 411, 170

Elmegreen, B. G. 1997, ApJ, 486, 944

Elmegreen, B. G., \& Parravano, A. 1994, ApJ, 435, L121

Erb, D. K., Steidel, C. C., Shapley, A. E., Pettini, M., Reddy, N. A., \& Adelberger, K. L. 2006, ApJ, 646, 107

Fry, A. M., Morrison, H. L., Harding, P., \& Boroson, T. A. 1999, AJ, 118, 1209

Gao, Y., \& Solomon, P. M. 2004, ApJ, 606, 271

Genzel, R., et al. 2010, MNRAS, 407, 2091

Gil de Paz, A., et al. 2007, ApJS, 173, 185

Gnedin, N. Y., \& Kravtsov, A. V. 2010, ApJ, 714, 287

González, V., Labbé, I., Bouwens, R. J., Illingworth, G., Franx, M., Kriek, M., \& Brammer, G. B. 2010, ApJ, 713, 115

Graciá-Carpio, J., Planesas, P., \& Colina, L. 2007, A\&A, 468, L67

Hainline, L. J., Blain, A. W., Smail, I., Alexander, D. M., Armus, L., Chapman, S. C., \& Ivison, R. J. 2010, arXiv:1006.0238

Helfer, T. T., Thornley, M. D., Regan, M. W., Wong, T., Sheth, K., Vogel, S. N., Blitz, L., \& Bock, D. C.-J. 2003, ApJS, 145, 259

Hinz, J. L., Rieke, M. J., Rieke, G. H., Willmer, C. N. A., Misselt, K., Engelbracht, C. W., Blaylock, M., \& Pickering, T. E. 2007, ApJ, 663, 895

Ho, L. C., Filippenko, A. V., \& Sargent, W. L. W. 1997, ApJ, 487, 591

Hollenbach, D. J., \& Tielens, A. G. G. M. 1999, Rev. Mod. Phys., 71, 173

Hopkins, P. F., Cox, T. J., Younger, J. D., \& Hernquist, L. 2009a, ApJ, 691, 1168

Hopkins, P. F., et al. 2009b, MNRAS, 397, 802

Hunter, D. A., \& Elmegreen, B. G. 2004, AJ, 128, 2170

Hunter, D. A., Elmegreen, B. G., \& Baker, A. L. 1998, ApJ, 493, 595

Irwin, J. A., Seaquist, E. R., Taylor, A. R., \& Duric, N. 1987, ApJ, 313, L91

Isobe, T., Feigelson, E. D., Akritas, M. G., \& Babu, G. J. 1990, ApJ, 364, 104

Jogee, S., Scoville, N., \& Kenney, J. D. P. 2005, ApJ, 630, 837

Karim, A., Schinnerer, E., VLA-COSMOS, T., \& COSMOS collaborations 2011, ApJ, 730, 61

Kelly, B. C. 2007, ApJ, 665, 1489

Kennicutt, R. C., Jr. 1989, ApJ, 344, 685

Kennicutt, R. C., Jr. 1998a, ApJ, 498, 541

Kennicutt, R. C., Jr. 1998b, ARA\&A, 36, 189

Kennicutt, R. C., Jr., et al. 2003, PASP, 115, 928

Kennicutt, R. C., Jr., et al. 2007, ApJ, 671, 333 
Kennicutt, R. C., et al. 2009, ApJ, 703, 1672

Knapp, G. R., \& Raimond, E. 1984, A\&A, 138, 77

Krumholz, M. R., McKee, C. F., \& Tumlinson, J. 2009, ApJ, 699, 850

Leroy, A. K., Walter, F., Brinks, E., Bigiel, F., de Blok, W. J. G., Madore, B., \& Thornley, M. D. 2008, AJ, 136, 2782

Maraston, C., Daddi, E., Renzini, A., Cimatti, A., Dickinson, M., Papovich, C., Pasquali, A., \& Pirzkal, N. 2006, ApJ, 652, 85

Maraston, C., Pforr, J., Renzini, A., Daddi, E., Dickinson, M., Cimatti, A., \& Tonini, C. 2010, MNRAS, 407, 830

Martin, C. L., \& Kennicutt, R. C., Jr. 2001, ApJ, 555, 301

Matthews, L. D., Gao, Y., Uson, J. M., \& Combes, F. 2005, AJ, 129, 1849

Neistein, E., \& Dekel, A. 2008, MNRAS, 383, 615

Noeske, K. G., et al. 2007a, ApJ, 660, L43

Noeske, K. G., et al. 2007b, ApJ, 660, L47

Oliver, S., et al. 2010, MNRAS, 405, 2279

Oosterloo, T., et al. 2010, MNRAS, 409, 500

Papadopoulos, P. P., \& Pelupessy, F. I. 2010, ApJ, 717, 1037

Papovich, C., Finkelstein, S. L., Ferguson, H. C., Lotz, J. M., \& Giavalisco, M. 2011, MNRAS, 412, 1123

Paturel, G., Petit, C., Prugniel, P., Theureau, G., Rousseau, J., Brouty, M., Dubois, P., \& Cambrésy, L. 2003, A\&A, 412, 45

Robertson, B., Bullock, J. S., Cox, T. J., Di Matteo, T., Hernquist, L., Springel, V., \& Yoshida, N. 2006, ApJ, 645, 986

Rodighiero, G., et al. 2010, A\&A, 518, L25

Roychowdhury, S., Chengalur, J. N., Begum, A., \& Karachentsev, I. D. 2009, MNRAS, 397, 1435

Ryder, S. D., \& Dopita, M. A. 1994, ApJ, 430, 142

Sage, L. J., \& Welch, G. A. 2006, ApJ, 644, 850

Schaerer, D., \& de Barros, S. 2010, A\&A, 515, A73

Schiminovich, D., et al. 2010, MNRAS, 408, 919

Schmidt, M. 1959, ApJ, 129, 243
Schuster, K. F., Kramer, C., Hitschfeld, M., Garcia-Burillo, S., \& Mookerjea B. 2007, A\&A, 461, 143

Scoville, N. Z., Yun, M. S., \& Bryant, P. M. 1997, ApJ, 484, 702

Sérsic, J. L., \& Pastoriza, M. 1967, PASP, 79, 152

Shapiro, K. L., et al. 2010, MNRAS, 402, 2140

Shi, Y., Rieke, G., Donley, J., Cooper, M., Willmer, C., \& Kirby, E. 2008, ApJ, 688, 794

Silk, J. 1997, ApJ, 481, 703

Solomon, P. M., Rivolo, A. R., Barrett, J., \& Yahil, A. 1987, ApJ, 319, 730

Springel, V., \& Hernquist, L. 2005, ApJ, 622, L9

Stark, D. P., Ellis, R. S., Bunker, A., Bundy, K., Targett, T., Benson, A., \& Lacy, M. 2009, ApJ, 697, 1493

Swinbank, A. M., et al. 2010, MNRAS, 405, 234

Tacconi, L. J., et al. 2008, ApJ, 680, 246

Thompson, T. A., Quataert, E., \& Murray, N. 2005, ApJ, 630, 167

van der Kruit, P. C., \& Searle, L. 1981, A\&A, 95, 105

van Driel, W., \& van Woerden, H. 1991, A\&A, 243, 71

Vázquez, G. A., \& Leitherer, C. 2005, ApJ, 621, 695

Walter, F., Brinks, E., de Blok, W. J. G., Bigiel, F., Kennicutt, R. C., Thornley, M. D., \& Leroy, A. 2008, AJ, 136, 2563

Wei, L. H., Vogel, S. N., Kannappan, S. J., Baker, A. J., Stark, D. V., \& Laine, S. 2010, ApJ, 725, L62

Wong, T., \& Blitz, L. 2002, ApJ, 569, 157

Wu, J., Evans, N. J., II, Gao, Y., Solomon, P. M., Shirley, Y. L., \& Vanden Bout, P. A. 2005, ApJ, 635, L173

Wyder, T. K., et al. 2009, ApJ, 696, 1834

Yabe, K., Ohta, K., Iwata, I., Sawicki, M., Tamura, N., Akiyama, M., \& Aoki, K. 2009, ApJ, 693, 507

Yun, M. S., \& Scoville, N. Z. 1995, ApJ, 451, L45

Yun, M. S., Scoville, N. Z., \& Knop, R. A. 1994, ApJ, 430, L109

Zheng, X. Z., Bell, E. F., Papovich, C., Wolf, C., Meisenheimer, K., Rix, H.-W., Rieke, G. H., \& Somerville, R. 2007, ApJ, 661, L41 